\title{
A TWO PRESSURE NUMERICAL MODEL OF TWO FLUID MIXING*
}

\author{
JAMES GLIMM ${ }^{\dagger}$, HYEONSEONG JIN`, MARC LAFOREST ${ }^{\ddagger}$, FOLKERT TANGERMAN ${ }^{\ddagger}$ \\ AND YONGMIN ZHANG $\ddagger$
}

\begin{abstract}
We present a numerical model of two fluid mixing based on hyperbolic equations having complete state variables (velocity, pressure, temperature) for each fluid. The model is designed for the study of acceleration driven mixing layers in a chunk mix regime dominated by large scale coherent mixing structures. The numerical solution of the model is validated by comparison to the incompressible limit. For the purpose of this comparison, we present a newly obtained analytic solution of the pressure equation for this model and an analytic constraint derived from the asymptotic limit of the compressible pressures, which determines uniquely the incompressible pressure solution. The numerical solution is also validated by a mesh convergence study.
\end{abstract}

Key words. multiphase flow, Rayleigh-Taylor instability

AMS subject classifications. 76T10, 76F50, 37M05

DOI. $10.1137 / \mathrm{S} 1540345903408464$

\section{Introduction.}

1.1. Fluid mixing. Fluid mixing is important to technology, for example in pipeline flow and the performance of inertial confinement fusion reactors; it is important to the description of natural phenomena, as in meteorological temperature inversion driven updrafts and the geological evolution of salt dome formations; it is important to basic science in the evolution of supernova explosions. Fluid mixing is a multiscale phenomenon, with active length scales from the submillimeter microscale of diffusive mixing to laboratory, geophysical, or astrophysical scales of macroscopic flow. It is neither possible nor desirable to simulate all fine details accurately. There are neither the computer resources nor the initialization data to allow resolution of fine details, while for many purposes statistical averages at a macroscopic level are sufficient and offer a more understandable description of the multifluid behavior. For this reason we seek averaged or coarse grained equations to describe the flow behavior of a multifluid mixture without direct simulation of all its microscopic details.

From the point of view of averaged equations, a multifluid mixture is a complex fluid with extra internal degrees of freedom. Starting from hyperbolic microscopic equations of two immiscible fluids, we consider averaged equations which are also hyperbolic, and which thus have only real characteristic speeds. The derivation of averaged equations leads to undefined averages of products or nonlinear functions of

*Received by the editors May 28, 2002; accepted for publication (in revised form) May 14, 2003; published electronically July 17, 2003. This work was supported in part by U.S. Department of Energy grants DE-FG02-90ER25084, DE-FG02-98ER25363, DE-FG03-98DP00206, and DE-AC0298CH10886, National Science Foundation grant DMS-0102480, Army Research Office grants DAAD190110642 and DAAL-03910027, and Los Alamos National Laboratory.

http://www.siam.org/journals/mms/1-3/40846.html

${ }^{\dagger}$ Center for Data Intensive Computing, Brookhaven National Laboratory, Upton, NY 11793-6000. Current address: Department of Applied Mathematics and Statistics, University at Stony Brook, Stony Brook, NY 11794-3600 (glimm@ams.sunysb.edu).

${ }^{\ddagger}$ Department of Applied Mathematics and Statistics, University at Stony Brook, Stony Brook, NY 11794-3600 (hjin@ams.sunysb.edu, laforest@ams.sunysb.edu, tangerman@ams.sunysb.edu, yzhang@ ams.sunysb.edu). The research of the third author was supported in part by Le Fonds pour la Formation de Chercheurs et l'Aide à la Recherche (FCAR), Canada. 
the primitive variables. These quantities must be modeled, i.e., expressed approximately as a function of the averaged flow quantities directly, to close the system of equations. Closure is a physics level identity, independent of the averaged governing equations. It restricts the applicability of the resulting system to the specific flow regime for which this identity has an approximate experimental validity. Multifluid flows possess a number of distinct flow regimes, which call for distinct closures, further complicating their study.

We are concerned with chunk mix [6,9], a term we have introduced to describe a flow regime characterized by large scale coherent mixing structures (bubbles of light fluid, etc.), on the order of the thickness of the mixing zone, and by short time scales so that relaxation terms are omitted $[3,26,27,29,6]$. Alternate models and closures are presented in $[41,17,44]$. Averaged flow models differ primarily in the order of statistical moments carried as dependent solution variables (we use a first moment closure), in the completeness of the multiphase treatment (we use a fully multiphase formulation in that no fluid variables are shared or averaged in common between the phases), and in the choice of specific closures, which usually correspond to some specification of the flow regime.

The closure used here is based on an assumed absence of internal length scales within the mixing zone, which is a mixing zone homogeneity assumption. It assumes independent thermodynamic variables (pressures and temperatures) for each phase, in addition to independent velocities. Its applicability includes rapid acceleration driven mixing processes for a chunk flow regime. For this purpose, we omit relaxation source terms which equilibrate pressures and velocities between the phases. We refer the reader to [6] and the references therein for more information on this model and its closure assumptions.

The main result of this paper is the presentation in section 2 of a numerical algorithm for the solution of the chunk mix multiphase fluid flow model with explicit tracking of the interface between the pure and multifluid regions. In section 3 we find a closed form solution for the incompressible one-dimensional (1D) pressure of this model, thereby completing the closed form solution of the incompressible 1D equations [28].

The model depends on the motions $Z_{k}(t)$ of the edges of the mixing zone. Since the $Z_{k}$ are not well characterized for compressible flows, we explore the dependence of the solution on the $Z_{k}$. In fact, most of the solution variables, suitably scaled, are nearly independent of the $Z_{k}$. This is not the case for the pressure differences. These differences appear in the exact (momentum) equation for the edge motion. In section 4.1, this fact is further related to the modeling of the drag and added mass terms in phenomenological buoyancy drag equations for the edge motion. The connection of the pressure difference to key terms in these buoyancy drag equations provides a physical basis for the sensitive dependence of the pressure difference on the edge motion.

Comparison to the analytic solution allows a numerical study of the incompressible limit for compressible flows, as a validation test of the numerical algorithm presented here. See section 4.2. Validation of the algorithm is also performed in section 4.3 through mesh convergence studies. Averaging of nonlinear equations, even the averaging of equations in conservation form, often leads to nonconservative equations. The present equations are an example. For such equations, the mesh refinement required for accurate solution of the equations is an important consideration. We show mesh convergence beginning at a mesh level having between eight cells (at the problem 
initialization) to 25 cells (at the time used for mesh convergence) within the mixing zone. A two-dimensional (2D) simulation is presented in section 4.3.

1.2. A two pressure two fluid flow model. We are concerned with the dynamic growth of a thin mixing layer formed at the interface between two fluids and embedded in a larger flow field. Random perturbations on the interface grow due to fluid instabilities and evolve into a complex flow microstructure to give rise to the mixing zone. We are here concerned with acceleration driven mixing. The averaging process used to define the model equations can be thought of as a local spatial or temporal average, or as an ensemble average, with respect to an ensemble of random initial conditions.

Within each realization the evolution of the interface is chaotic; at time $t$, the same space point may be occupied by fluid 1 or fluid 2, depending upon the position of the interface in that realization. Several realizations lead to the consideration of a mixed zone of fluids where, on average, one finds fluid $k$ at a given point $p$ only a fraction $\beta_{k}=\beta_{k}(t, p)$ of the ensemble total. Clearly, $\beta_{k}=1$ corresponds to a pure fluid region, where there is no mixture at all, a situation that happens away from the interface of all realizations of the ensemble. Evidently,

$$
\beta_{1}+\beta_{2}=1 \text {. }
$$

We parameterize the fluids by the discrete index $k, k=1,2$, and let $\beta_{k}, \rho_{k}, \vec{v}_{k}$, $p_{k}$, and $e_{k}$ be the volume fraction, density, velocity, pressure, and total energy of fluid $k$, respectively. The model we study is defined by the system of equations

$$
\begin{aligned}
\partial_{t} \beta_{k}= & -\vec{v}^{*} \cdot \nabla \beta_{k}, \\
\partial_{t} \rho_{k}+\nabla \cdot\left(\rho_{k} \vec{v}_{k}\right)= & -\rho_{k} \frac{\left(\vec{v}_{k}-\vec{v}^{*}\right)}{\beta_{k}} \cdot \nabla \beta_{k}, \\
\partial_{t}\left(\rho_{k} \vec{v}_{k}\right)+\nabla \cdot\left(\rho_{k} \vec{v}_{k} \otimes \vec{v}_{k}\right)+\nabla p_{k}= & -\rho_{k} \vec{v}_{k} \frac{\left(\vec{v}_{k}-\vec{v}^{*}\right)}{\beta_{k}} \cdot \nabla \beta_{k} \\
& -\frac{\left(p_{k}-p^{*}\right)}{\beta_{k}} \nabla \beta_{k}+\rho_{k} \vec{g}, \\
\partial_{t}\left(\rho_{k} e_{k}\right)+\nabla \cdot\left(\rho_{k} e_{k} \vec{v}_{k}\right)+\nabla \cdot\left(p_{k} \vec{v}_{k}\right)= & -\rho_{k} e_{k} \frac{\left(\vec{v}_{k}-\vec{v}^{*}\right)}{\beta_{k}} \cdot \nabla \beta_{k} \\
& +\frac{\left((p \vec{v})^{*}+p^{*} \vec{v}_{k}-2 p_{k} \vec{v}_{k}\right)}{\beta_{k}} \cdot \nabla \beta_{k} \\
& +\rho_{k} \vec{v}_{k} \cdot \vec{g},
\end{aligned}
$$

proposed in $[3,26,27,29,6]$. Here, $\vec{g}$ is the gravity and $\vec{v}^{*}, p^{*}$, and $(p \vec{v})^{*}$ represent averages to be modeled by a closure expression,

$$
\begin{aligned}
\vec{v}^{*} & =\mu_{1}^{v} \vec{v}_{2}+\mu_{2}^{v} \vec{v}_{1}, \\
p^{*} & =\mu_{1}^{p} p_{2}+\mu_{2}^{p} p_{1}, \\
(p \vec{v})^{*} & =\mu_{1}^{p} p_{2} \vec{v}_{2}+\left(1-\mu_{1}^{p}-\mu_{2}^{v}\right) p_{1} \vec{v}_{2}+\mu_{2}^{v} p_{1} \vec{v}_{1} .
\end{aligned}
$$

The coefficients $\mu_{k}^{v}$ and $\mu_{k}^{p}$ can be proved quite generally to define convex sums so that $\mu_{1}^{q}+\mu_{2}^{q}=1, \mu_{k}^{q} \geq 0, q=v, p[28]$. These coefficients are assumed to depend on the volume fraction $\beta_{k}$ and $t$ only. Boundary conditions at the edge of the mixing zone imply

$$
\mu_{k}^{q}\left(t, \beta_{k}\right)=\frac{\beta_{k}}{\beta_{k}+d_{k}^{q}(t) \beta_{k^{\prime}}}, \quad q=v, p
$$


where $k^{\prime}=3-k$ is the complementary index to $k$ and the $d$ 's satisfy $d_{1}^{q} d_{2}^{q}=1$.

Restricting the model to one spatial dimension $z$ measuring height, let $z=Z_{k}(t)$ be the edge of the mixing zone where $\beta_{k}$ vanishes. As a convention, the pure fluid region for fluid 1 will be located above the pure fluid region for fluid 2 . Henceforth, we will assume that the mixing zone is expanding at both edges, i.e., $(-1)^{k} \dot{Z}_{k}(t) \geq 0$, and set $V_{k}=\left|\dot{Z}_{k}(t)\right|$. In one spatial dimension, the constitutive factor $d_{k}^{v}$ was shown [33] to be a $z$-dependent ratio of volume creation terms for the two multiphase species, independent of any closure assumptions. As a condition of closure, we assume $d_{k}^{v}$ is independent of $z$ and obtain

$$
d_{k}^{v}(t)=\frac{\int_{Z_{k}}^{Z_{k^{\prime}}} \frac{\partial v_{k^{\prime}}}{\partial z}+\frac{1}{\rho_{k^{\prime}}} \frac{D_{k^{\prime}} \rho_{k^{\prime}}}{D t} d z}{\int_{Z_{k}}^{Z_{k^{\prime}}} \frac{\partial v_{k}}{\partial z}+\frac{1}{\rho_{k}} \frac{D_{k} \rho_{k}}{D t} d z} .
$$

Refer to [24]. Here $D_{k} / D t \equiv \partial / \partial t+v_{k} \partial / \partial z$ denotes the phase $k$ convective derivative. The formula (6) defines the constitutive law as a function of the solution variables. In the weakly compressible limit, a perturbative analysis $[33,24]$ is applied to (6). This analysis supplies new information on incompressible pressures, which appear in second order in the Mach number in this expansion. From this analysis, we see [33] that underdetermination of the incompressible pressure is resolved by information supplied from the weakly compressible theory. The choice for the pressure closure

$$
d_{k}^{p}=\rho_{k^{\prime}} / \rho_{k}
$$

appears quite naturally.

The system (1) is missing one condition at each edge $z=Z_{k}(t)$ of the mixing zone. Each missing condition is associated with a missing characteristic at the $Z_{k}$ boundary. For the fluid with vanishing $\beta_{k}$, the sonic characteristic entering from the $\beta_{k}=0$ side is missing. This missing information is supplied by the edge acceleration, $\ddot{Z}_{k}(t)$.

Thus we regard the edge positions $Z_{k}(t)$ as input, or data, which complete the specification of the model or close it. We appeal to the buoyancy drag model (see [29] and references therein) to provide the $Z_{k}(t)$. In this sense we separate and almost totally decouple the complete multiphase model into distinct edge and interior models, with the edge model completing the closure of the entire model. Many authors do not decouple this analysis and consider logically linked edge and interior equations. To the authors, the separation of these two distinct issues into two distinct models appears to be advantageous or at least harmless. With the mixing zone edge accelerations given and constitutive laws (6) and (7), the model has no adjustable parameters.

The mixing zone edge information will be supplied through a phenomenological buoyancy drag ODE to specify the edge accelerations,

$$
(-1)^{k} \frac{d^{2} Z_{k}(t)}{d t^{2}}=A g-\frac{\rho_{k^{\prime}}}{\rho_{k}+\rho_{k^{\prime}}} C_{k} \frac{V_{k}^{2}}{\left|Z_{k}\right|},
$$

where the Atwood number $A=\frac{\rho_{2}-\rho_{1}}{\rho_{2}+\rho_{1}}$. Equations of the general form (8) are known as buoyancy drag equations and have been considered by a number of authors [31, $1,7,14,38]$. A systematic error $[1,14]$ has been the use of the displacing fluid density $\rho_{k}$ in the numerator of the last term of (8). See [36] for correct discussion of 
drag. This error was corrected in [7] and subsequently in [38]. In the absence of this correction, a singular drag coefficient is required for $A=1$. Given (8) and the selfsimilar solution $Z_{k}=(-1)^{k} \alpha_{k} A g t^{2}$ for steady acceleration (Rayleigh-Taylor) mixing, there is a unique, one to one correspondence between $C_{k}$ and $\alpha_{k}$. For example,

$$
\begin{aligned}
C_{1} & =\frac{1 / \alpha_{1}-2}{2(1+A)}, \\
\alpha_{1} & =\frac{1}{2\left[1+C_{1}(1+A)\right]}
\end{aligned}
$$

so that with $\alpha_{1}=0.06, C_{1}$ varies by a factor of 2 from $C_{1}(A=0)=9$ to $C_{1}(A=1)=$ 4.5. We fit the available data on $\alpha_{1}$, which does not show a significant trend in its dependence on $A$, and regard (9) as a definition of $C_{1}$. See the appendix for the $\alpha_{1}$ data.

The buoyancy drag equations (8) used to specify the edge equations depend parametrically on the Atwood number, and thus on the local density field. For (nearly) incompressible flow, the Atwood number can be specified from $t=0$ data, and in this case the edge equations (buoyancy drag) and interior equations (1) decouple. In the general (compressible) case, the boundary Atwood number depends on the solution of the interior equations so that the coupling between the edge equations and the interior is weak but nonzero.

The vertical length $\left|Z_{k}\right|$ in (8) is replaced in [38] with a horizontal length, the wave length $\lambda$. For this reason, the evaluation of $\alpha_{1}$ in terms of $C_{1}$ in [38] depends on an additional ratio: the bubble height to bubble width ratio to convert a horizontal to a vertical length; this ratio, however, is not needed in [7]. The treatment of [38] assumes that the drag coefficient $C_{1} \equiv 2 \pi$ is independent of the Atwood number leading to a calculated value of $\alpha_{1}$ varying from $\alpha_{1}=0.065$ at $A=0$ to $\alpha_{1}=0.05$ at $A=1$. After adjustment in [8] for the factor $\lambda /\left|Z_{1}\right|=3$ so that $\lambda$ appears in the denominator of the decay term (8), $C_{1}$ in [7] varies by a factor of 2 , from 3 for $A=0$ to 1.5 for $A=1$, assuming $\alpha_{1}=0.05$. The experimental data shows no clear trend in the $A$ dependence of $\alpha_{1}$ so that a constant $\alpha_{1}$ appears to be a better interpretation of the experimental facts than is a constant $C_{1}$. The theoretically determined [8] value for $\lambda /\left|Z_{1}\right|=3$ lies in the middle of the experimental values, whereas the value $\lambda /\left|Z_{1}\right|=1.5$ of [38] is outside the experimental range. For this reason the drag coefficients from [7] and [38] do not agree.

We now describe the specific configuration to feature in our analysis and simulation. Suppose a slab of heavy fluid of density $\rho_{2}$ lies beneath a slab of light fluid of density $\rho_{1}<\rho_{2}$, separated by an interface. This configuration is then accelerated downwards with an acceleration larger than the earth gravity, effectively reversing the direction of gravity. In the following, the fluids occupy a container $[0,1] \times[-1,1]$ with periodic boundary conditions in the horizontal direction. We impose zero velocity conditions at the upper boundary $z=1.0$ and constant pressure conditions at the lower boundary $z=-1.0$ for our incompressible calculations. For the compressible calculations, we impose a rigid wall at the top, $z=1.0$, and outflow boundary conditions connecting to a fixed ambient state at $z=-1.0$. The $2 \mathrm{D}$ simulation in section 4.3 is given by a long wave length perturbation of this slab geometry.

2. The numerical algorithm. The algorithm presented in this section is the extension of the front tracking algorithm $[20,21,23,22,25,30]$ to the two fluid mixture model (1). The mixing zone edges $Z_{k}(t)$ needed to close the model can be used without transverse spatial dependence. 
Front tracking is a numerical method in which selected waves are explicitly represented in the discrete form of the solution. Examples include shock waves, contact discontinuities, and material interfaces. Other waves, such as leading and trailing edges of rarefaction waves, have continuous states but have jumps in their first derivatives. Tracked waves are propagated using the appropriate equations of motion for the given model. For example, if the system of equations consists of a set of hyperbolic conservation laws, $\mathbf{u}_{t}+\nabla \cdot \mathbf{f}=\mathbf{h}$, then the instantaneous velocity $s$ of a discontinuity surface satisfies the Rankine-Hugoniot equations, $s[\mathbf{u}]=[\mathbf{f}] \cdot \mathbf{n}$. Here $\mathbf{n}$ is the unit normal to the discontinuity surface. During a time step propagation, the type of a wave and the flow field in a neighborhood of the wave determine a local time integrated velocity for each point on the wave in the direction normal to the wave front. Wave propagation consists of moving each point a distance $s \Delta t$ in the normal direction as well as computing the time updated states at the new position. Tracking preserves the mathematical structure of the discontinuous waves by maintaining the discrete jump at the wave front, thus eliminating numerical diffusion. It also allows for the direct inclusion of the appropriate flow equations for the wave front in the numerical solution. The stencils used for the update of the interior states, which are irregular due to overlap with the front, are extended with ghost cells [25] to consist of state values from only one side of the front.

A tracked wave, known as a front (or, in two dimensions, a curve), is a piecewise linear representation of a physical wave, which is embedded in the underlying finite difference rectangular grid. A curve (or front) is a set of piecewise linear segments, called bonds. Each bond connects two points located at its two ends. Stored with each point are two states to represent the discontinuity across the wave. An orientation is given along the curve so that we may speak of the left and right states at a point. Propagation of the front can be defined as updating the position of each point on the front and updating the corresponding left and right states associated with it at a new time $t+\Delta t$. Operator splitting, in the rotated tangential normal coordinate system $(\vec{T}, \vec{N})$, allows separate propagation steps in directions normal to and tangent to the front. The tangential propagation is performed on each side of the front followed by the normal propagation. Since the solution is smooth during the tangential propagation, a convenient finite difference scheme, such as Lax-Wendroff, can be used to update the states at each point on the front. Notice that tangential propagation of points on the front is equivalent to remeshing of the front, in the limit $\Delta t \rightarrow 0$, so it is not essential to move these points during the tangential update.

On the computational domain we find a fixed rectangular computational grid and an independently gridded lower-dimensional front. This front is comprised of the mixing zone edges. Its mesh size is of the order of magnitude of the spacing in the rectangular grid. The complement of the front consists of either single phase or two phase fluids. Fluid states are assumed to vary smoothly within the closure of each component.

The data for the numerical solution consist of a (single or two phase) fluid state attached to every gridblock center, representing the average fluid state in the block and a (single or two phase) fluid state at each of the two sides of the front at any front grid point. These data, together with the choice of an interpolation method, determine the (single or two phase) fluid state at any point in any component, and thus the numerical solution.

2.1. Propagation of front points. Point propagation is a basic front tracking operation; for the gas dynamical version, see [10]. This operator computes the time- 
advanced position and state of the front. The currently implemented algorithm uses local-dimensional splitting to decompose the equations of motion into components normal and tangential to the interface. Let $\vec{N}, \vec{T}$ be the normal and tangential unit vectors at some point on the front. Then $\overrightarrow{v_{k}}$ can be rewritten as $\vec{v}_{k}=v_{k}^{N} \vec{N}+v_{k}^{T} \vec{T}$, $\overrightarrow{v^{*}}=v_{N}^{*} \vec{N}+v_{T}^{*} \vec{T}$, and $\vec{g}=g_{N} \vec{N}+g_{T} \vec{T}$. The projection of the equations (1) onto the normal direction can be written as

$$
\begin{aligned}
\partial_{t} \beta_{k}= & -v_{N}^{*} \frac{\partial \beta_{k}}{\partial N}, \\
\partial_{t} \rho_{k}+\frac{\partial \rho_{k} v_{k}^{N}}{\partial N}= & -\rho_{k} \frac{\left(v_{k}^{N}-v_{N}^{*}\right)}{\beta_{k}} \frac{\partial \beta_{k}}{\partial N}, \\
\partial_{t}\left(\rho_{k} v_{k}^{N}\right)+\frac{\partial}{\partial N}\left(\rho_{k} v_{k}^{N} v_{k}^{N}\right)+\frac{\partial p_{k}}{\partial N}= & -\rho_{k} v_{k}^{N} \frac{\left(v_{k}^{N}-v_{N}^{*}\right)}{\beta_{k}} \frac{\partial \beta_{k}}{\partial N} \\
& -\frac{\left(p_{k}-p^{*}\right)}{\beta_{k}} \frac{\partial \beta_{k}}{\partial N}+\rho_{k} g_{N}, \\
\partial_{t}\left(\rho_{k} v_{k}^{T}\right)+\frac{\partial}{\partial N}\left(\rho_{k} v_{k}^{T} v_{k}^{N}\right)= & -\rho_{k} v_{k}^{T} \frac{\left(v_{k}^{N}-v_{N}^{*}\right)}{\beta_{k}} \frac{\partial \beta_{k}}{\partial N}, \\
\partial_{t}\left(\rho_{k} e_{k}\right)+\frac{\partial}{\partial N}\left(\rho_{k} e_{k} v_{k}^{N}\right)+\frac{\partial}{\partial N}\left(p_{k} v_{k}^{N}\right)= & -\rho_{k} e_{k} \frac{\left(v_{k}^{N}-v_{N}^{*}\right)}{\beta_{k}} \frac{\partial \beta_{k}}{\partial N} \\
& +\frac{\left(\left(p v_{N}\right)^{*}+p^{*} v_{k}^{N}-2 p_{k} v_{k}^{N}\right)}{\beta_{k}} \frac{\partial \beta_{k}}{\partial N} \\
& +\rho_{k} v_{k}^{N} g_{N} \cdot
\end{aligned}
$$

Figure 1 shows the basic stencil of states used to compute the contribution to the normal component of flow. The states $s l_{0}$ and $s r_{0}$ denote the left and right states at the point to be propagated. States $s l_{i}$ and $s r_{i}$ are interpolated at distances $\Delta n$ in the direction normal to the front. The projection of these states onto the line normal to the front is used to compute the interface velocity at the point and a pair of time updated left and right states at the front.

The system (11) is hyperbolic, with eigenvalues $\lambda^{*}=v_{N}^{*}, \lambda_{k}^{ \pm}=v_{k}^{N} \pm c_{k}$, and $\lambda_{k}^{0}=v_{k}^{N}$ corresponding, respectively, to characteristics $C^{*}, C_{k}^{ \pm}$, and $C_{k}^{0}$. The $\lambda_{k}^{0}$ eigenvalue has multiplicity two, arising in both the tangential velocity and energy equations in (11). Here $c_{k}$ is a macroscopic sound speed. The characteristic equations of the system (11) are

$$
\begin{array}{rl}
\left(d \beta_{k}\right)_{C^{*}} & =0, \\
\left(\xi_{k}^{0} d \beta_{k}-c_{k}^{2} d \rho_{k}+d p_{k}\right)_{C_{k}^{0}} & 0, \\
\left(d v_{k}^{T}\right)_{C_{k}^{0}} & =0, \\
\left(\xi_{k}^{ \pm} d \beta_{k} \pm \rho_{k} c_{k}\left(d v_{k}^{N}-g_{N} d t\right)+d p_{k}\right)_{C_{k}^{ \pm}} & 0,
\end{array}
$$

with

$$
\begin{aligned}
\xi_{k}^{0} & =\frac{W_{k}-\rho_{k} c_{k}^{2}\left(v_{k}^{N}-v_{N}^{*}\right)}{\beta_{k}\left(v_{k}^{N}-v_{N}^{*}\right)}, \\
\xi_{k}^{ \pm} & =\frac{W_{k} \pm c_{k}\left(p_{k}-p^{*}\right)}{\beta_{k}\left(v_{k}^{N} \pm c_{k}-v_{N}^{*}\right)}, \\
W_{k} & =\left(\gamma_{k}-1\right)\left[\rho_{k} \epsilon_{k}\left(v_{k}^{N}-v_{N}^{*}\right)+p_{k} v_{k}^{N}-\left(p v_{N}\right)^{*}\right] .
\end{aligned}
$$



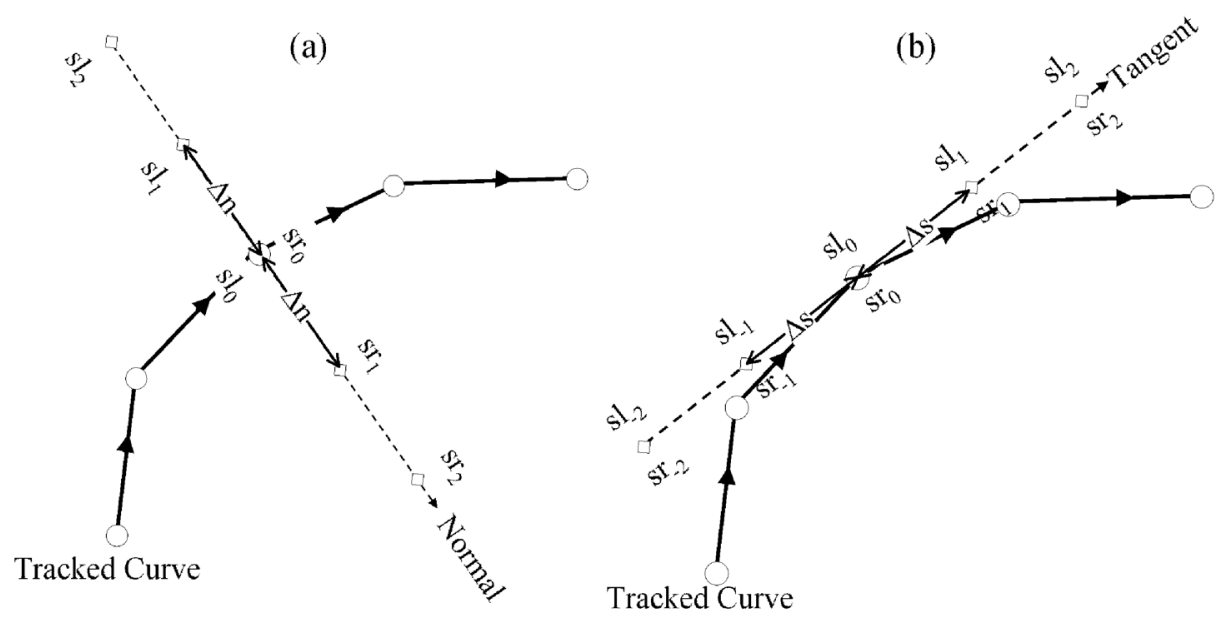

FIG. 1. A schematic showing the stencil of states used in propagating a front point. For simplicity the diagram is shown for two space dimensions. The normal propagate stencil is shown in (a), while (b) shows the stencil used in the tangential update.

Let $Z_{1}^{n}$ be the bubble (vanishing light fluid) mixing zone edge position, $Z_{2}^{n}$ be the spike (vanishing heavy fluid) mixing zone edge position, and $\left(v_{1}^{N}\right)_{01}^{n},\left(v_{2}^{N}\right)_{02}^{n}$ be the normal velocities of fluid 1 and fluid 2 at the corresponding positions $Z_{1}^{n}$ and $Z_{2}^{n}$ at time $t^{n}$. The edge position $Z_{k}^{n+1}$ at the next time level $t^{n+1}$ is updated using

$$
Z_{k}^{n+1}=Z_{k}^{n}+\left(v_{k}^{N}\right)_{0 k}^{n} \vec{N}_{k} \Delta t, \quad k=1,2,
$$

where $\vec{N}_{1}$ and $\vec{N}_{2}$ are normal vectors at $Z_{1}^{n}$ and $Z_{2}^{n}$, respectively.

The heavy fluid is continuous across the bubble edge, and the light fluid is continuous across the spike edge. The continuous phase states on the front are updated by interpolation from interior states after the interior update. Here we describe the update of vanishing phase on the front by applying the method of characteristics and the buoyancy drag law.

First, we update the normal component of the vanishing phase velocity $\left(v_{k}^{N}\right)_{0 k}$ on the edge $k$ by finite differencing (8) and noting that $\left(v_{k}^{N}\right)_{0 k}$ is the same as the edge normal velocity $\dot{Z}_{k}(t)=V_{k}(t)$ :

$$
\left(v_{k}^{N}\right)_{0 k}^{n+1}=\left(v_{k}^{N}\right)_{0 k}^{n}+(-1)^{k} \Delta t\left\{A_{0 k}^{n} g-C_{k}^{d}\left[\frac{\left(\rho_{k}^{\prime}\right)_{0 k}^{n}}{\left(\rho_{2}\right)_{0 k}^{n}+\left(\rho_{1}\right)_{0 k}^{n}}\right] \frac{\left(v_{k}^{N}\right)_{0 k}^{n}\left(v_{k}^{N}\right)_{0 k}^{n}}{\left(Z_{k}\right)^{n}}\right\},
$$

where $A_{0 k}^{n}$ is the instantaneous Atwood ratio at edge $k, A_{0 k}^{n}=\frac{\left(\rho_{2}\right)_{0 k}^{n}-\left(\rho_{1}\right)_{0 k}^{n}}{\left(\rho_{2}\right)_{0 k}^{n}+\left(\rho_{1}\right)_{0 k}^{n}}$. For the tangential velocity update, we have

$$
\left(v_{k}^{T}\right)_{0 k}^{n+1}=\left(v_{k}^{T}\right)_{0 k}^{n}
$$

due to (14).

Second, we use characteristic differencing to update the pressure at the bubble edge $(k=1)$ by tracing back the $C_{1}^{-}$characteristic from the space-time position 
$\left(Z_{1}^{n+1}, t_{n+1}\right)$ to $\left(z^{\prime}, t_{n}\right)$, with $z^{\prime}=Z_{1}^{n+1}-\left(v_{1}^{N}-c_{1}\right)_{01}^{n} \Delta t \vec{N}_{1}$. Note $z^{\prime}$ is located inside the mixing zone. Let $\left(U_{1}\right)^{\prime}$ be the phase 1 state at this space-time position $\left(z^{\prime}, t_{n}\right)$, found by interpolation on the numerical solution at time $t_{n}$. Approximating (15) by finite differences, we have

$$
\left(p_{1}\right)_{01}^{n+1}=p_{1}^{\prime}+\left(\xi_{1}^{-}\right)^{\prime} \beta_{1}^{\prime}+\left(\rho_{1} c_{1}\right)^{\prime}\left\{\left(v_{1}^{N}\right)_{01}^{n+1}-\left(v_{1}^{N}\right)^{\prime}-g \Delta t\right\} .
$$

At the spike edge $(k=2)$, we similarly obtain an approximation to (15) by tracing back the $C_{2}^{+}$characteristic from $\left(z_{2}^{n+1}, t_{n+1}\right)$ to $\left(z^{\prime}, t_{n}\right)$, with $z^{\prime}=Z_{2}^{n+1}-\left(v_{2}^{N}+c_{2}\right)_{02}^{n} \Delta t \vec{N}_{2}$ :

$$
\left(p_{2}\right)_{02}^{n+1}=p_{2}^{\prime}+\left(\xi_{2}^{+}\right)^{\prime} \beta_{2}^{\prime}-\left(\rho_{2} c_{2}\right)^{\prime}\left\{\left(v_{2}^{N}\right)_{02}^{n+1}-\left(v_{2}^{N}\right)^{\prime}-g \Delta t\right\} .
$$

Finally, we approximate (13) and obtain $\left(\rho_{k}\right)_{0 k}^{n+1}$ by noting $\left(\beta_{k}\right)_{0 k}^{n}=0$ for all $n$ :

$$
\left(\rho_{k}\right)_{0 k}^{n+1}=\left(\rho_{k}\right)_{0 k}^{n}+\frac{\left(p_{k}\right)_{0 k}^{n+1}-\left(p_{k}\right)_{0 k}^{n}}{\left(c_{k}^{2}\right)_{0 k}^{n}} .
$$

Note that there is no equation for the vanishing phase associated with the characteristic equations $C_{1}^{+}$and $C_{2}^{-}$at the two edges pointing out of the mixing zone. These equations are replaced by the buoyancy drag equation (8) at each edge so that the total number of equations is preserved.

2.2. Tangential sweep. Once front points have been processed using the normal point propagate operator, a second sweep is performed to incorporate tangential flow information. This is accomplished by projecting the states on a tracked wave near a point onto the tangent plane at the point being updated. As shown in Figure 1 for two space dimensions, states $s l_{i}$ and $s r_{i}$ are evaluated by interpolating points at distances $\Delta s$ in arclength along the curve, each taken from its respective side of the curve. The tangential projection of these states are then used as data for a standard finite difference solver that provides the final time updated state at the front point. The equations during the tangential sweep are given by

$$
\begin{aligned}
\partial_{t} \beta_{k}= & -v_{T}^{*} \frac{\partial \beta_{k}}{\partial T}, \\
\partial_{t} \rho_{k}+\frac{\partial \rho_{k} v_{k}^{T}}{\partial T}= & -\rho_{k} \frac{\left(v_{k}^{T}-v_{T}^{*}\right)}{\beta_{k}} \frac{\partial \beta_{k}}{\partial T}, \\
\partial_{t}\left(\rho_{k} v_{k}^{T}\right)+\frac{\partial}{\partial T}\left(\rho_{k} v_{k}^{T} v_{k}^{T}\right)+\frac{\partial p_{k}}{\partial T}= & -\rho_{k} v_{k}^{T} \frac{\left(v_{k}^{T}-v_{T}^{*}\right)}{\beta_{k}} \frac{\partial \beta_{k}}{\partial T} \\
& -\frac{\left(p_{k}-p^{*}\right)}{\beta_{k}} \frac{\partial \beta_{k}}{\partial T}+\rho_{k} g_{T}, \\
\partial_{t}\left(\rho_{k} v_{k}^{N}\right)+\frac{\partial}{\partial T}\left(\rho_{k} v_{k}^{N} v_{k}^{T}\right)= & -\rho_{k} v_{k}^{N} \frac{\left(v_{k}^{T}-v_{T}^{*}\right)}{\beta_{k}} \frac{\partial \beta_{k}}{\partial T}, \\
\frac{\partial}{\partial T}\left(\rho_{k} e_{k} v_{k}^{T}\right)+\frac{\partial}{\partial T}\left(p_{k} v_{k}^{T}\right)= & -\rho_{k} e_{k} \frac{\left(v_{k}^{T}-v_{T}^{*}\right)}{\beta_{k}} \frac{\partial \beta_{k}}{\partial T} \\
& +\frac{\left(\left(p v_{T}\right)^{*}+p^{*} v_{k}^{T}-2 p_{k} v_{k}^{T}\right)}{\beta_{k}} \frac{\partial \beta_{k}}{\partial T} \\
& +\rho_{k} v_{k}^{T} g_{T} \cdot
\end{aligned}
$$

The above equations are the projection of the system (1) onto the tangential direction $\vec{T}$ of the front. 
2.3. Interior update. Our approach to update the states in the interior region is to use the dimensional splitting method by decomposing the equations of motion into $x$ - and $y$-components and then to apply standard finite difference methods in each directional component. For example, our current implementation includes the LaxWendroff method $[37,40]$ and the MUSCL scheme $[11,2]$ in the $x$ - and $y$-directions.

The convergence of the splitting method is given by Crandall and Majda [12]. Strang [42] pointed out that the accuracy of splitting methods is second order if the order in which the $1 \mathrm{D}$ problems is solved is alternated, as in $x y y x x y \cdots$.

To couple the front information to the interior states, we consider the irregular finite difference stencils, which overlap or cross the tracked front. In updating such mesh blocks, we treat the tracked front as an internal time dependent "boundary." Missing stencil points on the "wrong" side of the interface are filled in by extrapolation as a ghost cell [25], using data from the same side of the interface as the mesh block being updated. The ghost cell method was introduced by Glimm, Marchesin, and McBryan in 1980 for this purpose. Therefore the front divides the domain into several subdomains. We solve the problem inside each subdomain with front states (from the correct front side) as boundary conditions. In that way, our finite differencing is never performed across the front, and the flow gradient remains perfectly sharp at the front.

3. Incompressible solutions. In this section we present an analytic solution for the incompressible pressure equations in the $1 \mathrm{D}$ case, which we use for the validation study in section 4 . This solution, of independent interest, completes the incompressible solution discussed in [6]. We also present an integral constraint on the incompressible pressures first derived in [33], which ensure their equality to the second term in the expansion of the compressible pressures in the incompressible limit. We emphasize the remarkable fact that the incompressible pressures, in the absence of a compressible limiting flow, are not unique. They are uniquely specified by certain details of the compressible flows from which they are derived as a limit.

3.1. Closed form incompressible pressures. The solutions for the incompressible pressures described in this section depend on earlier analytic solutions for the volume fractions and the velocities [6, 28]. These solutions for $\beta_{k}$ and $v_{k}$ are based on the observation that when the history of the mixing zone edges $Z_{k}(t)$ are known a priori, then the interface and continuity equations decouple from the momentum equations. This closed form solution for $\beta_{k}$ and $v_{k}$ is summarized in the following theorem.

Theorem 3.1. Consider incompressible flows in a finite but large interval $\boldsymbol{D}$ satisfying the closure models (2) and (5). Assume the boundary condition that the light velocity vanishes at the upper boundary of $\boldsymbol{D}$. Suppose that both edges $Z_{k}(t)$ are known and satisfy $V_{k} \equiv(-1)^{k} \dot{Z}_{k} \geq 0$. Suppose that $\beta_{k}$ is piecewise $C^{1}$ and initial profile $\beta_{k}(z, 0)$ is an invertible function of height $z$, and that $v_{k}$ is continuous across the mixing zone edge $Z_{k^{\prime}}$. Then the interface and continuity equations in (1) have a unique solution for $\beta_{k}$ and $v_{k}$. The velocity is given as a function of $\beta_{k}$ by

$$
v_{k}=\dot{Z}_{k} \mu_{k^{\prime}}^{v}\left(\beta_{k}, t\right)=\beta_{k^{\prime}} \dot{Z}_{k} \frac{V_{k^{\prime}}}{\bar{V}},
$$

where $\bar{V}=\beta_{1} V_{1}+\beta_{2} V_{2}$. The constitutive law $d_{k}^{v}$ in the model (5) is given by

$$
d_{k}^{v}(t)=\frac{V_{k^{\prime}}(t)}{V_{k}(t)}
$$


The volume fraction $\beta_{k}$ is given by the functional inverse of

$$
z\left(\beta_{k}, t\right)=z_{0}\left(\beta_{k}\right)+\int_{0}^{t} v^{*}\left(\beta_{k}, s\right) d s
$$

where $z_{0}\left(\beta_{k}\right)$ is the inverse of the initial volume fraction profile $\beta_{k}(z, 0)$.

Still under the assumptions of Theorem 3.1, we now describe analytic solutions for the pressures using $\beta_{k}$ and $t$ as the independent variables. We rewrite the momentum equation in (1) as

$$
\frac{\partial p_{k}}{\partial z}+(-1)^{k} \frac{p^{*}-p_{k}}{\beta_{k}} \frac{\partial \beta_{1}}{\partial z}=h_{k}(z, t)
$$

where

$$
h_{k}(z, t)=\rho_{k}\left(g-\frac{D_{k} v_{k}}{D t}\right)
$$

For any quantity $q$, set

$$
\bar{q} \equiv \beta_{1} q_{1}+\beta_{2} q_{2}, \quad \widetilde{q} \equiv q_{1}-d_{2}^{p} q_{2}, \quad \Delta q \equiv q_{1}-q_{2} .
$$

The linear combinations $\bar{p}$ and $\widetilde{p}$ decouple the momentum equations into a pair of ODEs:

$$
\frac{\partial \bar{p}}{\partial z}=\bar{h}, \quad \frac{\partial \widetilde{p}}{\partial z}=\widetilde{h} .
$$

This system can be integrated in closed form in terms of the history of the edge motions.

We begin by writing $h_{k}$ explicitly as a function of $\beta_{k}$ and $\dot{Z}_{k}$ and its derivatives. With (20) one can verify that

$$
\frac{\partial v_{k}}{\partial \beta_{k}}=(-1)^{k^{\prime}} \frac{V_{k}^{2} V_{k^{\prime}}}{\bar{V}^{2}}, \quad \frac{\partial v_{k}}{\partial V_{k}}=(-1)^{k} \frac{V_{k^{\prime}}^{2}}{\bar{V}^{2}} \beta_{k^{\prime}}^{2}, \quad \frac{\partial v_{k}}{\partial V_{k^{\prime}}}=(-1)^{k} \frac{V_{k}^{2}}{\bar{V}^{2}} \beta_{k^{\prime}} \beta_{k} .
$$

The $h_{k}$ terms in (25) contain convective derivatives that can be written in terms of $V_{k}$ and $\beta_{k}$. Let

$$
\zeta_{k} \equiv(-1)^{k} \ddot{Z}_{k} \frac{\partial v_{k}}{\partial V_{k}}+(-1)^{k^{\prime}} \ddot{Z}_{k^{\prime}} \frac{\partial v_{k}}{\partial V_{k^{\prime}}}=\frac{\beta_{k^{\prime}}}{\bar{V}^{2}}\left(\ddot{Z}_{k} V_{k^{\prime}}^{2} \beta_{k^{\prime}}-\ddot{Z}_{k^{\prime}} V_{k}^{2} \beta_{k}\right) .
$$

Then

$$
\begin{aligned}
\frac{\partial v_{k}}{\partial t} & =(-1)^{k} \frac{\partial v_{k}}{\partial V_{k}} \ddot{Z}_{k}+(-1)^{k^{\prime}} \frac{\partial v_{k}}{\partial V_{k^{\prime}}} \ddot{Z}_{k^{\prime}}+\frac{\partial v_{k}}{\partial \beta_{k}} \frac{\partial \beta_{k}}{\partial t} \\
& =\zeta_{k}-\frac{\partial v_{k}}{\partial \beta_{k}} \frac{\partial\left(\beta_{k} v_{k}\right)}{\partial z} \\
& =\zeta_{k}-\frac{\partial v_{k}}{\partial \beta_{k}}\left(v_{k}+\beta_{k} \frac{\partial v_{k}}{\partial \beta_{k}}\right) \frac{\partial \beta_{k}}{\partial z} .
\end{aligned}
$$

Substituting this result into $h_{k}$ and using (26), we obtain

$$
\begin{aligned}
h_{k} & =\rho_{k} g-\rho_{k} \zeta_{k}+\rho_{k}\left(\frac{\partial v_{k}}{\partial \beta_{k}}\left(v_{k}+\beta_{k} \frac{\partial v_{k}}{\partial \beta_{k}}\right) \frac{\partial \beta_{k}}{\partial z}-v_{k} \frac{\partial v_{k}}{\partial \beta_{k}} \frac{\partial \beta_{k}}{\partial z}\right) \\
& =\rho_{k} g-\rho_{k} \zeta_{k}+\rho_{k} \beta_{k}\left(\frac{\partial v_{k}}{\partial \beta_{k}}\right)^{2} \frac{\partial \beta_{k}}{\partial z} \\
& =\rho_{k} g-\rho_{k} \zeta_{k}+\rho_{k} \beta_{k} \frac{V_{k}^{4} V_{k^{\prime}}^{2}}{\bar{V}^{4}} \frac{\partial \beta_{k}}{\partial z} .
\end{aligned}
$$


Therefore, the right side of (25) is given explicitly as a function of $\beta_{k}$,

$$
\begin{aligned}
\bar{h}=\bar{\rho} g & +\frac{V_{1}^{2} V_{2}^{2}}{\bar{V}^{4}}\left(\beta_{1}^{2} \rho_{1} V_{1}^{2}-\beta_{2}^{2} \rho_{2} V_{2}^{2}\right) \frac{\partial \beta_{1}}{\partial z} \\
& +\left(\rho_{1}-\rho_{2}\right) \frac{\beta_{1} \beta_{2}}{\bar{V}^{2}}\left(\ddot{Z}_{2} V_{1}^{2} \beta_{1}-\ddot{Z}_{1} V_{2}^{2} \beta_{2}\right), \\
\widetilde{h}=\tilde{\rho} g & +\frac{V_{1}^{2} V_{2}^{2}}{\bar{V}^{4}}\left(\beta_{1} \rho_{1} V_{1}^{2}+\beta_{2} \rho_{2} d_{2}^{p} V_{2}^{2}\right) \frac{\partial \beta_{1}}{\partial z} \\
& +\frac{1}{\bar{V}^{2}}\left(\rho_{1} \beta_{2}+d_{2}^{p} \rho_{2} \beta_{1}\right)\left(\ddot{Z}_{2} V_{1}^{2} \beta_{1}-\ddot{Z}_{1} V_{2}^{2} \beta_{2}\right) .
\end{aligned}
$$

Observe that $\widetilde{\rho}=0$ if $d_{2}^{p}=\rho_{1} / \rho_{2}$ so that in this case $\widetilde{h}$ is independent of $g$.

The system (25) may be integrated with respect to $z$ since $\beta_{k}$ is a function of $z$. Instead, we rewrite the system in terms of $\beta_{1}$ since $\beta_{1}$ is expressed as an invertible function of $z$. We now transform these integrals into $\beta_{1}$ integrals. We differentiate $v^{*}=\mu_{2}^{v} v_{1}+\mu_{1}^{v} v_{2}$ with respect to $\beta_{k}$ using (20) to obtain

$$
\frac{\partial v^{*}}{\partial \beta_{k}}=2(-1)^{k^{\prime}} \frac{\dot{V}_{k^{\prime}}^{2} V_{k}^{2}}{\bar{V}^{3}} .
$$

This result is inserted into the derivative of (22) with respect to $\beta_{k}$. If we assume an infinitesimally thin initial mixing zone, then the term $z_{k}^{\prime}\left(\beta_{k}\right)=d z_{0} / d \beta_{k}$ in (22) vanishes and we obtain

$$
\frac{\partial z}{\partial \beta_{k}}=(-1)^{k^{\prime}} 2 \int_{0}^{t} \frac{V_{1}^{2}(s) V_{2}^{2}(s)}{\bar{V}^{3}(s)} d s .
$$

This identity reduces the integrals in $z$ to integrals in $\beta_{1}$.

3.1.1. Weakly self-similar solutions. Incompressible solutions that correspond to edge position functions of the form $Z_{k}(t)=(-1)^{k} \alpha_{k} Z(t)$ are called weakly self-similar. Define $V(t)=\dot{Z}(t)$.

The weak self-similar hypothesis allows evaluation of (30) as

$$
\frac{\partial z}{\partial \beta_{k}}=(-1)^{k^{\prime}} 2 \frac{\alpha_{1}^{2} \alpha_{2}^{2}}{\bar{\alpha}^{3}}\left(\int_{0}^{t} V(s) d s\right)=(-1)^{k^{\prime}} 2 \frac{\alpha_{1}^{2} \alpha_{2}^{2}}{\bar{\alpha}^{3}} Z(t),
$$

assuming $Z(0)=0$. As a result, the integral of $(25)$ can be written as an integral with respect to $\beta_{k}$. The integrand is a rational function of $\beta_{k}$ with a denominator which is a power of a linear function of $\beta_{k}$ times a polynomial in the instantaneous scale factor $Z(t)$ and its first and second derivatives. These integrals can be evaluated explicitly by a partial fraction decomposition, leading to the weakly self-similar pressure solutions

$$
\begin{aligned}
\bar{p}\left(\beta_{1}, t\right) & =p_{2}\left(Z_{1}\right)+\beta_{1} g Z(t) A_{0}+\beta_{1} V^{2}(t) B_{0}+\beta_{1} \Delta \rho \dot{V}(t) Z(t) C_{0} \\
A_{0} & =\frac{\alpha_{1}^{2}}{\bar{\alpha}^{2}}\left(\rho_{2} \bar{\alpha}+\bar{\rho} \alpha_{2}\right) \\
B_{0} & =\frac{\alpha_{1}^{2} \alpha_{2}}{3 \bar{\alpha}^{3}}\left[\beta_{1}^{2} \Delta \rho \alpha_{1}^{2}-3 \beta_{2} \rho_{2} \alpha_{2} \bar{\alpha}\right] \\
C_{0} & =\frac{\alpha_{1}^{3} \alpha_{2}}{3 \bar{\alpha}^{3}(\Delta \alpha)^{2}}\left[-2 \alpha_{1}\left(\bar{\alpha}^{2}+\bar{\alpha} \alpha_{2}+\alpha_{2}^{2}\right)+3 \bar{\alpha}\left(\alpha_{1}+\alpha_{2}\right)\left(\alpha_{2}+\bar{\alpha}\right)-6 \alpha_{2} \bar{\alpha}^{2}\right]
\end{aligned}
$$




$$
\begin{aligned}
\widetilde{p}\left(\beta_{1}, t\right) & =\widetilde{p}\left(Z_{1}\right)+\beta_{1} g \widetilde{\rho} Z(t) D_{0}+\beta_{1} V^{2}(t) E_{0}+\beta_{1} \dot{V}(t) Z(t) F_{0}, \\
D_{0} & =\frac{\alpha_{1}^{2}}{\bar{\alpha}^{2}}\left(\alpha_{2}+\bar{\alpha}\right) \\
E_{0} & =\frac{\alpha_{1}^{2}}{6 \bar{\alpha}^{3} \Delta \alpha}\left[-2 \alpha_{1} \widetilde{\rho \alpha}\left(\alpha_{2}^{2}+\alpha_{2} \bar{\alpha}+\bar{\alpha}^{2}\right)+3 \widetilde{\alpha} \widetilde{\rho \alpha^{2}}\left(\alpha_{2}+\bar{\alpha}\right)\right], \\
F_{0} & =\frac{\alpha_{1}^{3}}{3 \bar{\alpha}^{3} \Delta \alpha}\left[2 \widetilde{\rho \alpha}\left(\alpha_{2}^{2}+\alpha_{2} \bar{\alpha}+\bar{\alpha}^{2}\right)-3 \alpha_{2} \bar{\alpha} \widetilde{\rho}\left(\alpha_{2}+\bar{\alpha}\right)\right] .
\end{aligned}
$$

We recognize the $A_{0}$ and $D_{0}$ terms as buoyancy terms as they are multiplied by gravity. Similarly, the $B_{0}$ and $E_{0}$ terms, both proportional to $V^{2}$, are drag terms, while the $C_{0}$ and $F_{0}$ terms, proportional to $\ddot{Z}$, are inertial terms. The constitutive law closure (7) implies $\widetilde{\rho}=0$ so that the buoyancy contribution to $\widetilde{p}$ vanishes.

3.1.2. The general incompressible pressure solution. In general the identity (31) is not valid. Since the history integrals originating in (30) cannot be evaluated in closed form, they appear in the final expression for the pressures. We reverse the order of integration between $\beta_{1}$ and $t$. For fixed time $s$ in the history integral, the $\beta_{k}$ integrals are again evaluated in closed form by a partial fraction decomposition.

The analytic solutions for $\bar{p}$ and $\widetilde{p}$ are found by integrating these expressions. We concentrate on the solution for $\bar{p}$. Integrating the first equation of (28), we have

$$
\begin{aligned}
\bar{p}(z) & =\beta_{1} p_{1}(z)+\beta_{2} p_{2}(z)=\bar{p}\left(Z_{1}\right)+\int_{Z_{1}}^{z} \bar{h} d z \\
& =p_{2}\left(Z_{1}\right)+2 g \int_{0}^{t} V_{1}^{2}(s) V_{2}^{2}(s)\left[\int_{0}^{\beta_{1}} \frac{\rho_{1} \phi_{1}+\rho_{2} \phi_{2}}{\bar{V}^{3}(s)} d \phi_{1}\right] d s \\
& +V_{1}^{2}(t) V_{2}^{2}(t) \int_{0}^{\beta_{1}} \frac{\rho_{1} V_{1}^{2}(t) \phi_{1}^{2}-\rho_{2} V_{2}^{2}(t) \phi_{2}^{2}}{\bar{V}^{4}(t)} d \phi_{1} \\
& +2\left(\rho_{2}-\rho_{1}\right) \int_{0}^{t} V_{1}^{2}(s) V_{2}^{2}(s) \\
& \times\left[\int_{0}^{\beta_{1}} \frac{\phi_{1} \phi_{2}}{\bar{V}^{3}(s) \bar{V}^{2}(t)}\left(\ddot{Z}_{1} V_{2}^{2}(t) \phi_{2}-\ddot{Z}_{2} V_{1}^{2}(t) \phi_{1}\right) d \phi_{1}\right] d s .
\end{aligned}
$$

Note that all accelerations $\ddot{Z}_{k}=\ddot{Z}_{k}(t)$ are functions of time $t$ and that $\phi_{1}+\phi_{2}=1$. Analytic solutions can be obtained since the denominator involves only powers of $\bar{V}(s)$ and $\bar{V}(t)$, which are linear functions of $\beta_{k}$. We omit details concerning the integrations and partial fraction decompositions. All identities were found with the assistance of the computer algebra program Maple.

It is simple to check that

$$
\int_{0}^{\beta_{1}} \frac{\rho_{1} \phi_{1}+\rho_{2} \phi_{2}}{\bar{V}^{3}(s)} d \phi_{1}=\frac{1}{2} \frac{\beta_{1}}{V_{2}^{2}(s) \bar{V}^{2}(s)}\left(\rho_{2} \bar{V}(s)+\bar{\rho} V_{2}(s)\right) .
$$

Similarly for the second term, the integration with respect to $\beta_{1}$ is performed.

$$
\begin{aligned}
\int_{0}^{\beta_{1}} \frac{1}{\bar{V}^{4}(t)}\left(\rho_{1} V_{1}^{2}(t) \phi_{1}^{2}\right. & \left.-\rho_{2} V_{2}^{2}(t) \phi_{2}^{2}\right) d \phi_{1} \\
& =\frac{1}{3} \frac{\beta_{1}}{V_{2}(t) \bar{V}^{3}(t)}\left(\beta_{1}^{2} \Delta \rho V_{1}^{2}(t)-3 \beta_{2} \rho_{2} V_{2}(t) \bar{V}(t)\right) .
\end{aligned}
$$


The partial fraction decomposition for the last integrand in (33) gives rise to four different rational functions. We factor out the terms involving $\ddot{Z}_{k}$. Let $\eta=$ $V_{2}(t) V_{1}(s)-V_{1}(t) V_{2}(s)$.

$$
\begin{aligned}
\int_{0}^{\beta_{1}} & \frac{\phi_{1} \phi_{2}}{\bar{V}^{3}(s) \bar{V}^{2}(t)}\left(\ddot{Z}_{1} V_{2}^{2}(t) \phi_{2}-\ddot{Z}_{2} V_{1}^{2}(t) \phi_{1}\right) d \phi_{1} \\
= & -\frac{1}{\eta^{4}}\left(V_{2}^{3}(t) \ddot{Z}_{1}+V_{1}^{3}(t) \ddot{Z}_{2}\right) \\
& \times\left(\left(V_{2}(t) V_{1}(s)+V_{1}(t) V_{2}(s)\right) \ln \left(\frac{\bar{V}(t) V_{2}(s)}{\bar{V}(s) V_{2}(t)}\right)+\beta_{1} \eta\left(\frac{V_{1}(t)}{\bar{V}(t)}+\frac{V_{1}(s)}{\bar{V}(s)}\right)\right) \\
& -\frac{1}{\eta^{4}}\left(V_{2}^{2}(t) V_{2}(s) \ddot{Z}_{1}+V_{1}^{2}(t) V_{1}(s) \ddot{Z}_{2}\right)\left(V_{1}(t) V_{2}(t) \ln \left(\frac{\bar{V}(t) V_{2}(s)}{\bar{V}(s) V_{2}(t)}\right)\right. \\
& \left.-\frac{\beta_{1} V_{1}(s) \eta^{2}\left(V_{2}(s)+\bar{V}(s)\right)}{2 V_{2}(s) \bar{V}^{2}(s) \Delta V(s)}+\frac{\beta_{1} \eta\left(V_{2}(t) V_{1}^{2}(s)-V_{1}(t) V_{2}^{2}(s)\right)}{V_{2}(s) \bar{V}(s) \Delta V(s)}\right) .
\end{aligned}
$$

When these expressions are substituted into (33) we obtain

$$
\bar{p}(z)=p_{2}\left(Z_{1}\right)+\int_{Z_{1}}^{z} \bar{h} d z=p_{2}\left(Z_{1}\right)+\beta_{1} g A+\beta_{1} \bar{V}^{2}(t) B+2 \Delta \rho C,
$$

where

$$
\begin{gathered}
A=\int_{0}^{t} \frac{V_{1}^{2}(s)}{\bar{V}^{2}(s)}\left(\rho_{2} \bar{V}(s)+\bar{\rho} V_{2}(s)\right) d s \\
B=\frac{V_{1}^{2}(t) V_{2}(t)}{3 \bar{V}^{5}(t)}\left(\beta_{1}^{2} \Delta \rho V_{1}^{2}(t)-3 \beta_{2} \rho_{2} V_{2}(t) \bar{V}(t)\right) \\
C=\int_{0}^{t} \frac{V_{1}^{2}(s) V_{2}^{2}(s)}{\eta^{4}}\left[\left(V_{2}^{3}(t) \ddot{Z}_{1}(t)+V_{1}^{3}(t) \ddot{Z}_{2}(t)\right)\right. \\
\times\left(\left(V_{2}(t) V_{1}(s)+V_{1}(t) V_{2}(s)\right) \ln \left(\frac{\bar{V}(t) V_{2}(s)}{\bar{V}(s) V_{2}(t)}\right)+\beta_{1} \eta\left(\frac{V_{1}(t)}{\bar{V}(t)}+\frac{V_{1}(s)}{\bar{V}(s)}\right)\right) \\
+\left(V_{2}^{2}(t) V_{2}(s) \ddot{Z}_{1}(t)+V_{1}^{2}(t) V_{1}(s) \ddot{Z}_{2}(t)\right)\left(V_{1}(t) V_{2}(t) \ln \left(\frac{\bar{V}(t) V_{2}(s)}{\bar{V}(s) V_{2}(t)}\right)\right. \\
\left.\left.-\frac{\beta_{1} V_{1}(s) \eta^{2}\left(V_{2}(s)+\bar{V}(s)\right)}{2 V_{2}(s) \bar{V}^{2}(s) \Delta V(s)}+\frac{\beta_{1} \eta\left(V_{2}(t) V_{1}^{2}(s)-V_{1}(t) V_{2}^{2}(s)\right)}{V_{2}(s) \bar{V}(s) \Delta V(s)}\right)\right] d s .
\end{gathered}
$$

A similar analysis for $\widetilde{p}$ (details omitted) leads to

$$
\widetilde{p}(z)=\widetilde{p}\left(Z_{1}\right)+\int_{Z_{1}}^{z} \widetilde{h} d z=\widetilde{p}\left(Z_{1}\right)+\beta_{1} g \widetilde{\rho} D+\beta_{1} E+2 F
$$


where

$$
\begin{aligned}
& D=\int_{0}^{t} \frac{V_{1}^{2}(s)}{\bar{V}^{2}(s)}\left(V_{2}(s)+\bar{V}(s)\right) d s \\
& E=\frac{V_{1}^{2}(t)}{6 \bar{V}^{3}(t) \Delta V(t)} \\
& \times\left(-2 \widetilde{\rho V}(t) V_{1}(t)\left(V_{2}^{2}(t)+V_{2}(t) \bar{V}(t)+\bar{V}^{2}(t)\right)+3 \widetilde{\rho V^{2}}(t) \bar{V}(t)\left(V_{2}(t)+\bar{V}(t)\right)\right), \\
& F=\int_{0}^{t} \frac{V_{1}^{2}(s) V_{2}^{2}(s)}{\eta^{4}}\left[\left(V_{2}(s) V_{1}^{2}(t) \ddot{Z}_{2}(t)+V_{1}(s) V_{2}^{2}(t) \ddot{Z}_{1}(t)\right)\right. \\
& \times\left(2 \widetilde{\rho V}(t) \Delta V(t) \ln \left(\frac{\bar{V}(t) V_{2}(s)}{\bar{V}(s) V_{2}(t)}\right)+\frac{\beta_{1} \eta^{2}}{V_{2}(s) \bar{V}(s)}\left\{\frac{\widetilde{\rho V}(t) \Delta V(s)}{\eta}-\frac{\widetilde{\rho V}(s)\left(V_{2}(s)+\bar{V}(s)\right)}{2 V_{2}(s) \bar{V}(s)}\right\}\right) \\
& +\left(V_{1}(t) \ddot{Z}_{2}(t)+V_{2}(t) \ddot{Z}_{1}(t)\right) \\
& \times\left(2 \widetilde{\rho V^{2}}(t) \eta \ln \left(\frac{\bar{V}(t) V_{2}(s)}{\bar{V}(s) V_{2}(t)}\right)+\beta_{1} V_{1}(t) V_{2}(t) \eta\left\{\frac{\widetilde{\rho V}(t) \Delta V(t)}{V_{2}(t) \bar{V}(t)}+\frac{\widetilde{\rho V}(s) \Delta V(s)}{V_{2}(s) \bar{V}(s)}\right\}\right) \\
& \left.+\left(V_{1}(s) \ddot{Z}_{2}(t)+V_{2}(s) \ddot{Z}_{1}(t)\right) \widetilde{\rho V}(t) V_{1}(t) V_{2}(t) \Delta V(t) \ln \left(\frac{\bar{V}(t) V_{2}(s)}{\bar{V}(s) V_{2}(t)}\right)\right] d s .
\end{aligned}
$$

Again we recognize $A$ and $D$ as buoyancy terms, $B$ and $E$ as drag terms, and $C$ and $F$ as inertial terms. The parallelism of structure between the $A_{0}$ and $A$, etc. terms is evident. The buoyancy term $\beta_{1} g \widetilde{\rho} D$ vanishes due to (7). Observe that the $\bar{p}$ and $\widetilde{p}$ drag terms are instantaneous, i.e., have no history integral, while the buoyancy and inertial terms require history integrals.

3.2. Unique solvability of the pressure equation. The system (25) requires two independent boundary conditions. Assuming continuity of the pure to mixed fluid pressures, we have $\bar{p}\left(Z_{1}\right)=p_{2}\left(Z_{1}\right)$ and $\bar{p}\left(Z_{2}\right)=p_{1}\left(Z_{2}\right)$. However, these two values are not independent, as they are related by the identity

$$
\bar{p}\left(Z_{2}\right)=\bar{p}\left(Z_{1}\right)+\int_{Z_{1}}^{Z_{2}}\left(\beta_{1} h_{1}+\beta_{2} h_{2}\right) d z=\bar{p}\left(Z_{1}\right)+\int_{Z_{1}}^{Z_{2}} \bar{h} d z
$$

and so an additional condition is needed. This new relation is obtained by taking the incompressible limit of the compressible equations. The physical meaning of the incompressible pressure is quite different from that of the compressible case. Assume that the two compressible fluids are described by polytropic equations of state,

$$
p_{k}=\lambda^{2} A_{k} \rho_{k}^{\gamma_{k}}
$$

The entropy of fluid $k$ is given by $A_{k, \lambda}=\lambda^{2} A_{k}$. The incompressible limit is obtained as $\lambda \rightarrow \infty$. In this section, we assume isentropic flow so that the $A_{k}$ are constant. 
By [33], the state variables and constitutive parameters admit an expansion of the form

$$
\begin{aligned}
& \beta_{k}=\beta_{k}^{\infty}+O\left(\lambda^{-1}\right), \\
& v_{k}=v_{k}^{\infty}+O\left(\lambda^{-1}\right), \\
& \rho_{k}=\rho_{k}^{\infty}+O\left(\lambda^{-2}\right), \\
& p_{k}=\lambda^{2} p_{k}^{0}+p_{k}^{\infty}+O\left(\lambda^{-1}\right), \\
& d_{k}^{v}=d_{k}^{v \infty}+\lambda^{-1} d_{k}^{v(1, s)}+\lambda^{-2} d_{k}^{v(2, s)}+O\left(\lambda^{-3}\right),
\end{aligned}
$$

where the superscript $\infty$ indicates the incompressible solution and the exponent $(j, s)$, $j=1,2$, indicates the $j$ th order term for the slow variables in the expansion. Here $\lambda^{2} a_{k}^{2}=c_{k}^{2}\left(\rho_{k}^{\infty}\right)=\lambda^{2} A_{k} \gamma_{k} \rho_{k}^{\infty \gamma_{k}-1}$ is the leading order term for the sound speed squared in fluid $k$.

Note the quadratic leading order term in powers of $\lambda$ in the pressure. This term equals the pressure defined by the equation of state for fluid $k$, evaluated at $\rho_{k}^{\infty}$. Thus, $p_{k}^{0}=A_{k}\left(\rho_{k}^{\infty}\right)^{\gamma_{k}}$. Continuity of the compressible pressures at the interface requires $\lambda^{2} A_{1}\left(\rho_{1}^{\infty}\right)^{\gamma_{1}}=\lambda^{2} A_{2}\left(\rho_{2}^{\infty}\right)^{\gamma_{2}}$, and therefore

$$
A_{2}=\frac{\left(\rho_{1}^{\infty}\right)^{\gamma_{1}}}{\left(\rho_{2}^{\infty}\right)^{\gamma_{2}}} A_{1} .
$$

For incompressible flow, Theorem 3.1 gives the closure relation (21). Asymptotic analysis for slightly compressible flows shows that the relation (6) provides the equations

$$
\begin{aligned}
d_{1}^{v(1, s)}(t)= & \left(\frac{V_{2}}{V_{1}}\right)^{(1, s)}=\frac{V_{1}^{\infty} V_{2}^{(1, s)}-V_{2}^{\infty} V_{1}^{(1, s)}}{V_{1}^{\infty} 2}, \\
d_{1}^{v(2, s)}(t)= & \left(\frac{\int_{Z_{1}}^{Z_{2}} \frac{\partial v_{2}}{\partial z}+\frac{1}{\rho_{2}} \frac{D_{2} \rho_{2}}{D t} d z}{\int_{Z_{1}}^{Z_{2}} \frac{\partial v_{1}}{\partial z}+\frac{1}{\rho_{1}} \frac{D_{1} \rho_{1}}{D t} d z}\right)^{(2, s)} \\
= & \frac{-d_{1}^{v(1, s)} V_{1}^{(1, s)}-d_{1}^{v \infty} V_{1}^{(2, s)}+V_{2}^{(2, s)}}{V_{1}^{\infty}} \\
& -\frac{1}{V_{1}^{\infty}}\left\{\int_{Z_{1}^{\infty}}^{z^{+\infty}} \frac{\beta_{1}^{\infty}+d_{1}^{v \infty}}{\rho_{1}^{\infty} a_{1}^{2}}\left(\frac{\partial p_{1}^{\infty}}{\partial t}+v_{1}^{\infty} \frac{\partial p_{1}^{\infty}}{\partial z}\right) d z\right. \\
& \left.\quad+\int_{z^{-\infty}}^{Z_{2}^{\infty}} \frac{\beta_{2}^{\infty}-1}{\rho_{2}^{\infty} a_{2}^{2}}\left(\frac{\partial p_{2}^{\infty}}{\partial t}+v_{2}^{\infty} \frac{\partial p_{2}^{\infty}}{\partial z}\right) d z\right\}
\end{aligned}
$$

for the constitutive law $d_{k}^{v}$ through second order in the Mach number. Here $V_{k}^{(j, s)}=$ $\left|\dot{Z}_{k}^{(j, s)}(t)\right|, Z_{k}^{(j, s)}=Z_{k}^{(j, s)}(t)$ is the $j$ th order term in the expansion of the compressible mixing zone edge $Z_{k}$, and $z=z^{+\infty}\left(z=z^{-\infty}\right)$ is defined as the position of the upper (lower) wall of $\boldsymbol{D}$. Specifically, (43) resolves a degree of freedom in the incompressible pressures related to the relative compressibility of the two fluids. The incompressible pressures are uniquely determined by the limiting sound speeds $a_{k}$ of the two phases and the volume creation constitutive law $d_{1}^{v}$ in the weakly compressible $(2, s)$ limit. These relations and the convergence of the compressible multiphase solution to the incompressible solutions are studied in [33]. 
4. Simulation studies. Our numerical studies aim to validate the algorithm described in section 2 and explore the relationship between the model for edge motion (8) and our multiphase model inside the mixing zone. Both experiments are performed near the incompressible limit and therefore depend on the analysis of the compressible equations in the incompressible limit [33] and on the analytic solutions for this limit, both of which are summarized in section 3 .

We begin by reviewing the initialization of our model for acceleration driven fluid mixing. The two important parameters controlling the initialization used in section 4.1 and section 4.2 are the compressibility $\lambda$ (see (39) and (40)) and the drag coefficients $C_{k}$ in (8). The other parameters are adjusted to produce an initialization for the compressible code with few transients near the incompressible limit $\lambda \rightarrow \infty$.

The initialization proceeds as follows. For some dimensionless set of units, assume that the height $z$ of the container is parameterized by the interval $[-1,1]$. Suppose that the heavy fluid of density $\rho_{2}^{\infty}=0.4$ is located below the light fluid of density $\rho_{1}^{\infty}=0.1$ and that the container is given an upwards acceleration of $g=0.3$. The Atwood number is therefore $A \equiv\left(\rho_{2}^{\infty}-\rho_{1}^{\infty}\right) /\left(\rho_{1}^{\infty}+\rho_{2}^{\infty}\right)=0.6$. Each fluid is given a $\gamma$-law as an equation of state with $\gamma_{1}=1.4$ and $\gamma_{2}=1.8$ for, respectively, the light and heavy fluid.

According to self-similar edge motions of the incompressible flow,

$$
Z_{k}=(-1)^{k} \alpha_{k} A g t^{2},
$$

there remains only one parameter, the mixing growth rate $\alpha_{k}$, for the edges. According to formulas (9) and (10), the growth rates $\alpha_{k}$ are equivalent to the drag coefficients $C_{k}$. When $A \leq 0.9$, the hypothesis of a stationary center of mass [29] for the mixing zone is approximately valid [7]. This hypothesis implies an additional constraint, which determines the ratio

$$
\frac{\alpha_{2}}{\alpha_{1}}=\frac{-\frac{1}{3}\left(\rho_{2}^{\infty}-\rho_{1}^{\infty}\right)+\sqrt{\frac{1}{9}\left(\rho_{2}^{\infty}-\rho_{1}^{\infty}\right)^{2}+4 \rho_{1}^{\infty} \rho_{2}^{\infty}}}{2 \rho_{1}^{\infty}}
$$

of the mixing growth rates $\alpha_{k}$ as an exact identity [6] for incompressible flow. At the bubble edge, experimental results $[43,39,13,15,16]$ indicate that $\alpha_{1} \approx 0.06$, and by (45), $\alpha_{2}$ is fixed at $\alpha_{2}=0.093693$.

In order to have a reasonable number of grid points initially within the mixing zone, we set $Z_{2}\left(t_{0}\right)=0.1$ and begin the experiment at the time

$$
t_{0}=\sqrt{\frac{Z_{2}\left(t_{0}\right)}{\alpha_{2} A g}}
$$

after the beginning of the expansion of the mixing zone. This value $t_{0}$ is then used with (45) to obtain the initial position of $Z_{1}\left(t_{0}\right)$ and the velocities $\dot{Z}_{k}\left(t_{0}\right), k=1,2$ :

$$
\begin{aligned}
& Z_{1}\left(t_{0}\right)=-0.06403882, \\
& \dot{Z}_{1}\left(t_{0}\right)=v_{1}\left(Z_{1}\left(t_{0}\right), t_{0}\right)=-V_{1}\left(t_{0}\right)=-0.05259731, \\
& \dot{Z}_{2}\left(t_{0}\right)=v_{2}\left(Z_{2}\left(t_{0}\right), t_{0}\right)=V_{2}\left(t_{0}\right)=0.08213348 .
\end{aligned}
$$

The initial state for compressible multiphase flow $\left(\beta_{k}(0), \rho_{k}(0), v_{k}(0), p_{k}(0)\right)$ is 
defined as

$$
\begin{aligned}
& \beta_{k}(0)=\beta_{k}^{\infty}\left(t_{0}\right) \\
& v_{k}(0)=v_{k}^{\infty}\left(t_{0}\right) \\
& \rho_{k}(0)=\rho_{k}^{\infty}\left(t_{0}\right) \\
& p_{k}(0)=\lambda^{2} p_{k}^{0}+p_{k}^{\infty}\left(t_{0}\right)
\end{aligned}
$$

according to (40).

4.1. Model dependence on edge motions. The equation

$$
\begin{aligned}
(-1)^{k} \rho_{k} \frac{d^{2} Z_{k}(t)}{d t^{2}}= & \left(\rho_{2}-\rho_{1}\right) g+\frac{\partial\left(p_{1}-p_{2}\right)}{\partial z} \\
& -\rho_{k^{\prime}} V_{k}^{2} \frac{\partial \beta_{1}}{\partial z}-(-1)^{k}\left(d_{k^{\prime}}^{p}-1\right)\left(p_{1}-p_{2}\right) \frac{\partial \beta_{1}}{\partial z}
\end{aligned}
$$

has been derived [28] as an exact consequence of the momentum equation for incompressible flow. Here the right-hand side of this equation is evaluated along the trajectory $z=Z_{k}(t)$. Newton's law (48) of acceleration relates the phenomenological drag coefficient and added mass in (8) to the pressure difference and the gradient of the pressure difference. These latter quantities, determined in closed form for incompressible flow in (37)-(38), thus allow a derivation of (8) with a theoretical determination of added mass and drag. The interpretation of the separate terms in (37)-(38) is discussed in section 3.1. The structure of these terms is complicated, and a simple interpretation of the connection between (48), (8), and (37)-(38) is outside the scope of the present work. However, we make a few qualitative observations, based on the numerical solution of the chunk mix multiphase equations (1).

In (48) the acceleration term is positive for both $k=1$ and $k=2$. The gravity term $\left(\rho_{2}-\rho_{1}\right) g$ is positive and thus increases acceleration, while the drag term proportional to $-V_{k}^{2}$, being negative, always decelerates. Assuming $d_{k}^{p}=\rho_{k^{\prime}} / \rho_{k}$, the coefficient $-(-1)^{k}\left(d_{k^{\prime}}^{p}-1\right) \partial \beta_{1} / \partial z$ of the pressure difference is always nonpositive. Since $p_{1}-p_{2}$ is positive in Figures 4 and 5 , this term in (48) is a deceleration term for the fluid parameters considered here. The pressure difference gradient, generally negative for the edge motions shown here, is also a deceleration term.

From the above analysis, we can understand the dependence of the multiphase solution on the edge motions $Z_{k}(t)$. The $Z_{k}$ are determined through specification of the drag coefficient $C_{k}$. Since the dependence of the $C_{k}$ on compressibility is not understood, we explore the dependence of the solution on the $C_{k}$. The influence of a variation in $C_{k}$ on the multiphase solutions can most easily be understood through its influence on $\alpha_{k}$ via (10). We plot normalized variables with this direct effect removed. Figure 2 (left) indicates that the quantity $\beta_{k}$ plotted in terms of a normalized height variable is insensitive to changes in the mixing growth rates $\alpha_{k}$ (determined by the drag coefficient $C_{k}(t)$ ). The edge velocity scaling (linear growth in time) is complicated due to an initial time offset in the amount $t_{0}$ and by the use of an incompressible initialization for the compressible dynamical evolution. Thus we introduce the scaling velocity

$$
\mathcal{V}_{k}(t)=2 \alpha_{k}^{0} A g t_{0}+2 \alpha_{k} A g t \approx(-1)^{k} V_{k}(t),
$$

where $\alpha_{k}^{0}=\alpha_{k}\left(C_{k}^{0}\right)$ and $\alpha_{k}\left(C_{k}\right)$ are defined by (10) and $t \geq 0$. From Figure 2 (right), we see that $v_{k} / \mathcal{V}_{k}$ is insensitive to the choice of compressible drag coefficients. In 

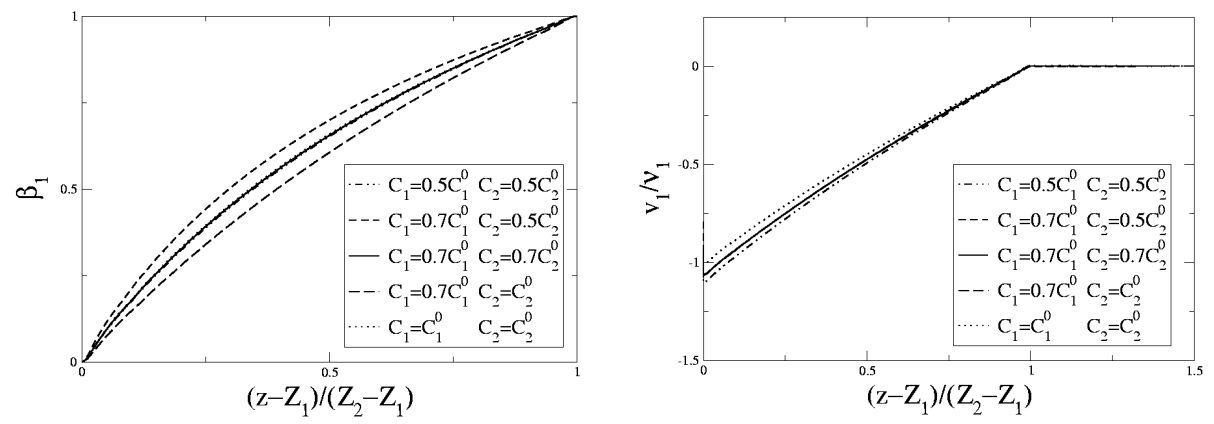

FIG. 2. The insensitivity of the volume fraction and scaled velocity to the drag coefficients $C_{k}$ and the resulting mixing zone edges $Z_{k}$ when $\lambda^{2}=25$ and $t=3.0$. These variables, when plotted vs. a scaled height $\left(z-Z_{1}\right) /\left(Z_{2}-Z_{1}\right)$, are nearly indistinguishable for a considerable variation in the $Z_{k}$. Here $C_{k}^{0}$ is the incompressible drag coefficient.

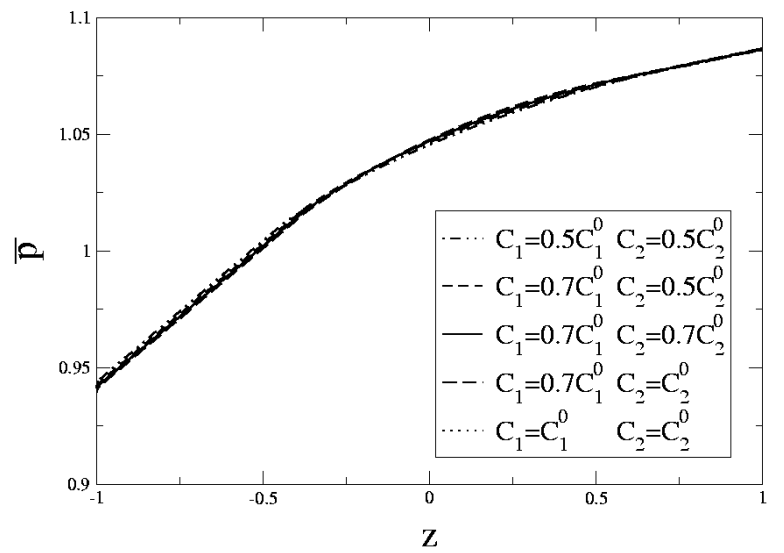

FIG. 3. Insensitivity of the mean pressure $\bar{p}$ to variation of the drag coefficients and the resulting edge motions $Z_{k}$. Here $\bar{p}$ is plotted vs. $z$ and the other parameters are as in Figure 2.

fact the small remaining dependence on the compressible drag coefficients in Figure 2 (right) is due to the fact that $V_{k}(t) / \mathcal{V}_{k}(t)$ is not exactly equal to $(-1)^{k}$. See the left edge of Figure 2 (right), where $v_{k}\left(Z_{k}\right) / \mathcal{V}_{k}=V_{k} / \mathcal{V}_{k} \approx(-1)^{k}$. A plot of $v_{k} / V_{k}$ vs. $z /\left(Z_{2}-Z_{1}\right)$ (not shown) displays no visible dependence on the drag coefficient. Similarly, $\bar{p}$, plotted vs. $z$ in Figure 3 , is insensitive. The variation in the drag coefficient considered here corresponds to $30 \%$ and $70 \%$ increments in the edge motions (and $\alpha$ 's), relative to the incompressible edge motions determined by the incompressible drag coefficients $C_{k}^{0}$.

However, in Figure 4, the scaled pressure difference and its gradient, especially at the spike edge of the mixing zone, are seen to be sensitive to the drag coefficients and thus to the edge motions they determine. As the edges move faster (i.e., as the drag coefficient is decreased), the pressure difference at the spike edge responds with an increase in $\left(p_{1}-p_{2}\right) /\left(Z_{2}-Z_{1}\right)$ (increased deceleration) and in $\nabla\left(p_{1}-p_{2}\right)$ 


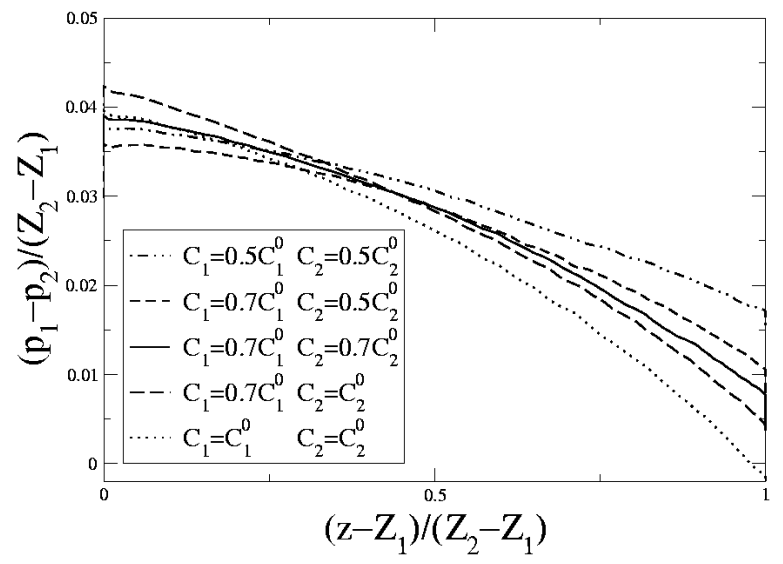

FIG. 4. The sensitivity of the pressure difference for the compressible equations to the drag coefficients and resulting mixing zone edges $Z_{k}$ when $\lambda^{2}=25$ and $t=3.0$. We plot $\left(p_{1}-p_{2}\right) /\left(Z_{2}-Z_{1}\right)$ vs. $\left(z-Z_{1}\right) /\left(Z_{2}-Z_{1}\right)$ for several choices of drag coefficients $C_{k}$ and corresponding growth rate coefficients $\alpha_{k}=\alpha_{k}\left(C_{k}\right)$.
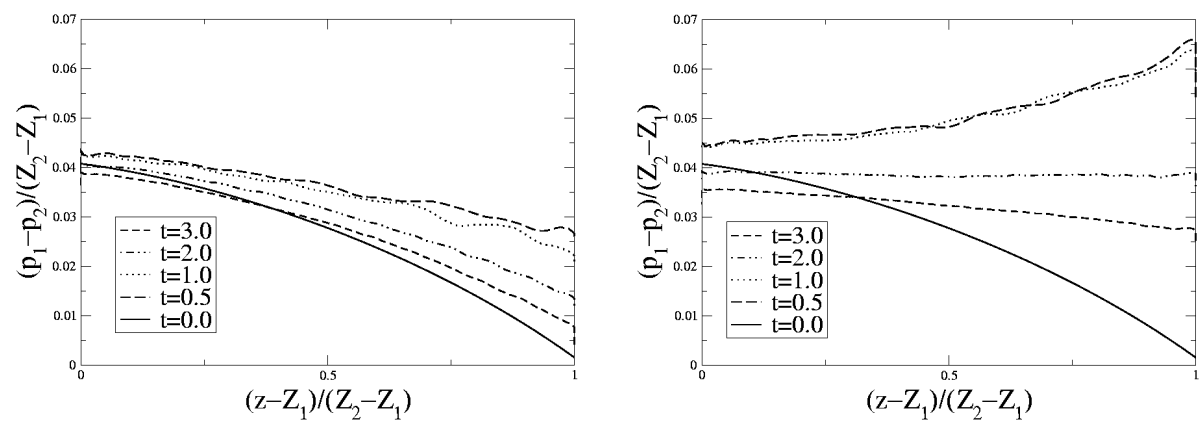

FIG. 5. The pressure difference depends on time. Displayed are the pressure difference curves for $t=0.0,0.5,1.0,2.0,3.0$. Left: $\lambda^{2}=25$ (nearly incompressible), Right: $\lambda^{2}=5$ (moderately compressible).

(decreased deceleration). Thus we see that the modification in the acceleration is accommodated by a change in the balance between the contribution of these two forces. Surprisingly, this balance is sensitive to a change in either the bubble or the spike drag coefficient. Between these two terms at the bubble edge, the gradient term appears to be more significant at the spike edge. At the bubble edge, the gradient of the pressure difference is nearly zero, and the pressure difference induced drag results mainly from the pressure difference term. This term scales directly with the change in edge positions. Thus we see little competition between the two terms, and the one term which plays an active role varies in direct proportion to the effect causing this change. The present discussion applies only to the Atwood number $(A=0.6)$ considered here and does not fully explore the role of these two pressure difference terms in fixing the value for the phenomenological drag coefficient. 
Initialization transients occur in the balance between the two pressure difference terms. After the transients have died out, the pressure difference resembles its incompressible value qualitatively, and this late time pressure difference approaches the analytically determined value in the incompressible limit. See Figure 5. In the nearly incompressible left frame, about 50 sound wave crossings have occurred, and initialization transients are small for the final time shown, $t=3.0$. In the more compressible right frame, only about 10 sound speed crossings have occurred. Thus there has been less time for the transients to decay in the right frame. Moreover, the incompressible initialization used here will generate larger transients in the more compressible right frame. For single phase Euler equations, it has been proved by the uniform analysis that a more compressible system is more oscillatory in early time and that the oscillatory waves decay after a certain small time $[34,35,32]$. This fact is not guaranteed for the multiphase flow equations. The complete analysis of the oscillatory part of solutions for the multiphase flow equations has not been reported yet. In Figure 5 the $t=0.5$ and $t=1.0$ curves of the scaled pressure difference involve initialization transients rather than oscillatory waves.

We recall that the nonuniqueness of the incompressible solution [28] is required to accommodate distinct incompressible limits for the pressure difference. Specifically the incompressible pressure difference depends on the ratios of compressible sound speeds (compressibilities) and compressible corrections to the edge motion $Z_{k}(t)$. The latter depend on the compressibilities, the hydrostatic temperature stratification, and compressibility corrections to the buoyancy drag equation (such as a dependence of the drag on the compressibility). For this reason, we believe the explicit dependence on the edge motions will turn out to be a strength, not a weakness, of the multiphase equations (1) in allowing the full range of solutions physically achievable.

4.2. Validation. We validate our code via convergence under mesh refinement and convergence of the compressible solutions to the incompressible limit given in section 3 as $\lambda \rightarrow \infty$.

4.2.1. Convergence to the incompressible limit. The incompressible limit of the single phase compressible Euler equations has been studied theoretically in higher space dimensions $[18,34,35]$. The multiphase flow case, in contrast, presents additional difficulties so that even 1D multiphase flow is nontrivial. We rely here on the 1D multiphase flow analysis with a uniformly valid asymptotic expansion obtained previously [33]. The difference $p_{1}-p_{2}$ between the pressures of the two fluids is the most sensitive of the primitive variables in the equations, as we see when studying convergence in the incompressible limit.

The incompressible limit is difficult to achieve numerically within a compressible computational framework due to the short time steps (large sound speed) and possible oscillatory behavior, which are characteristic of compressible simulations. As shown in [33] the solutions generally decompose into a slow expansion part with a slow time scale and an oscillatory part with a fast time scale. The oscillatory component is evidence of rapid equilibration, which complicates the numerical convergence to the incompressible limit. Our use of the incompressible limit solution for initialization reduces the fast time scale oscillations. While fast variable oscillations are of interest, we have largely eliminated them from simulations presented in this paper.

In studying the limit $\lambda \rightarrow \infty$, we set $C_{k}=C_{k}^{0}$ for $\lambda^{2}=100, C_{k}=0.7 C_{k}^{0}$ for $\lambda^{2}=25, C_{k}=0.5 C_{k}^{0}$ for $\lambda^{2}=10$, and $C_{k}=0.3 C_{k}^{0}$ for $\lambda^{2}=5$. Figures 6 and 7 display convergence of the volume fraction $\beta_{1}$ and the scaled velocities $v_{k} / \mathcal{V}_{k}$ to the incompressible limit as $\lambda \rightarrow \infty$ for $\lambda^{2}=100,25,10,5$. Again, use of a scaled velocity 


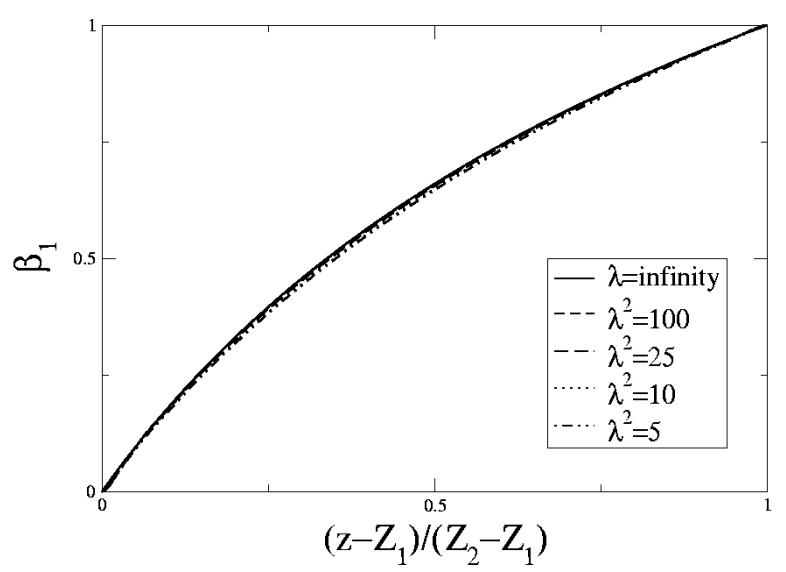

FIG. 6. Convergence of the volume fraction of light fluid to the analytical incompressible solution at $t=3.0$ as $\lambda \rightarrow \infty$.
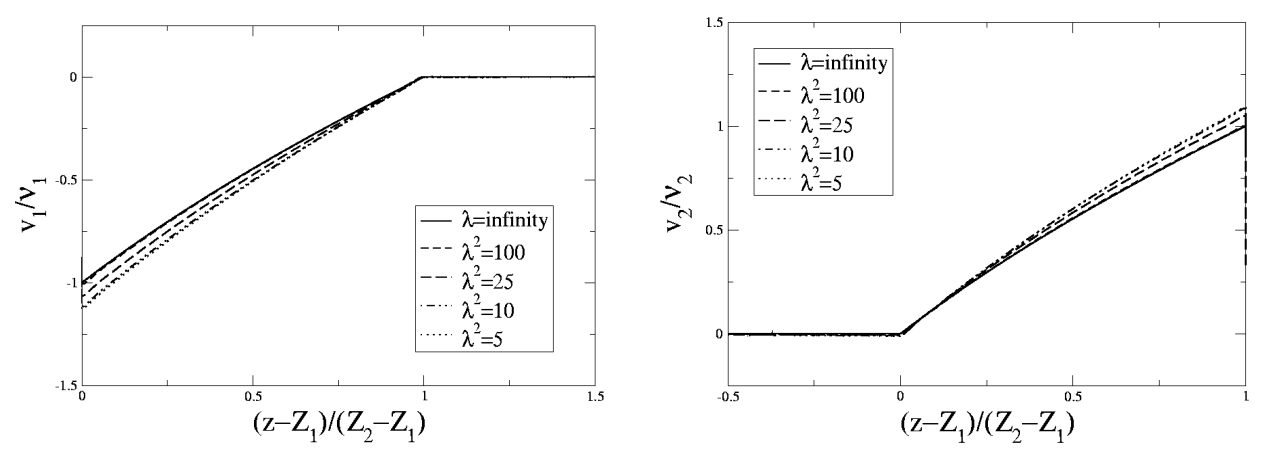

FIG. 7. Convergence of the scaled velocities to the analytical incompressible solution as $\lambda \rightarrow \infty$. Here $t=3$.0. Left: light (upper) fluid, Right: heavy (lower) fluid.

$v_{k} / V_{k}$ would accelerate the convergence. We plot the curves $p_{1}-p_{2}$ and $p_{1}^{\infty}-p_{2}^{\infty}$ for $t=3.0$ in Figure 8 for $\lambda^{2}=100,25,10,5$. This figure establishes the convergence of the pressure difference to the incompressible solution as $\lambda \rightarrow \infty$. Note that the $\lambda^{2}=100$ curve has crossed over the $\lambda=\infty$ curve in this limit. Due to the illconditioned nature of the incompressible limit when computed with a compressible code (as has been observed in some calculations not reported here), we do not believe the $\lambda^{2}=100$ calculation in Figure 4 has sufficient accuracy to attach significance to this cross over. As shown in Figure 4, the pressure difference is sensitive to the growth rates of compressible mixing zone, while Figures 2 and 3 show that the volume fraction, velocities, and mean pressure are insensitive. In particular, the pressure difference at the spike edge, even after scaling by $\left(Z_{2}-Z_{1}\right)$, depends on the bubble and spike growth rates.

4.2.2. Convergence under mesh refinement. We conduct the simulations for $\lambda^{2}=100$ with grid sizes of $100,200,500$, and 1000 . The physical parameters are 


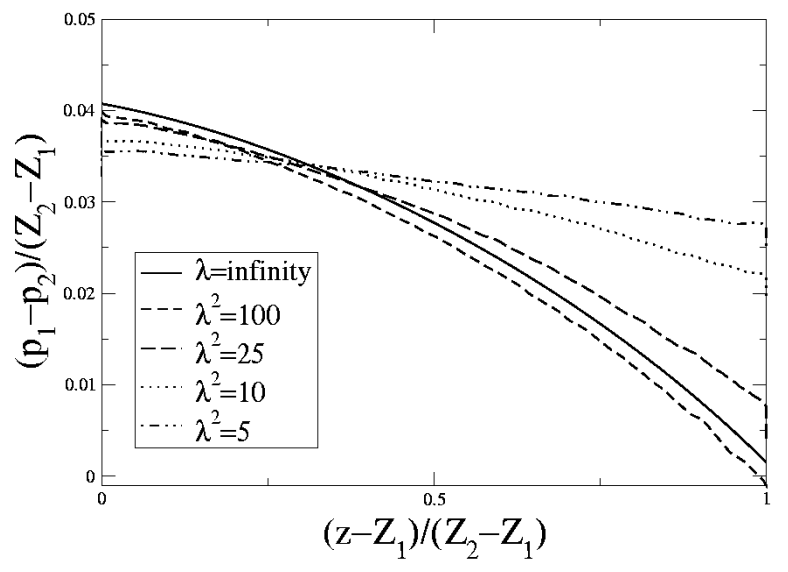

FIG. 8. Convergence of the pressure difference within the mixing zone to the analytical incompressible solution as $\lambda \rightarrow \infty$. Here $t=3.0$.

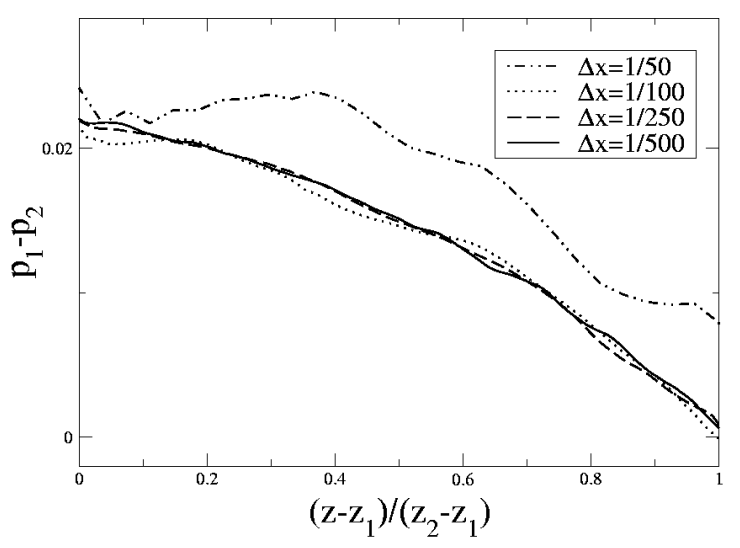

FIG. 9. The convergence of the pressure difference within the mixing zone under mesh refinement. Here $\lambda^{2}=100$ and $t=2.0$.

the same as those described in section 4.1. We set $C_{k}=C_{k}^{0}$.

At time $t=2$ the mixing zone comprises $1 / 4$ of the computational domain so that the coarsest $\Delta x=1 / 50$ mesh, for example, has 25 cells within the mixing zone. Figure 9 shows the convergence of the pressure difference within the mixing zone under mesh refinement. The convergence of two fluid velocities under mesh refinement is demonstrated in Figure 10. Since the plots of volume fraction and density are almost indistinguishable for different grid sizes, we omit them here. For the coarsest grid level shown here, the number of grid cells within the mixing zone increases from eight to 25 over the coarse of the simulation. Note that the volume fraction, velocities, and 

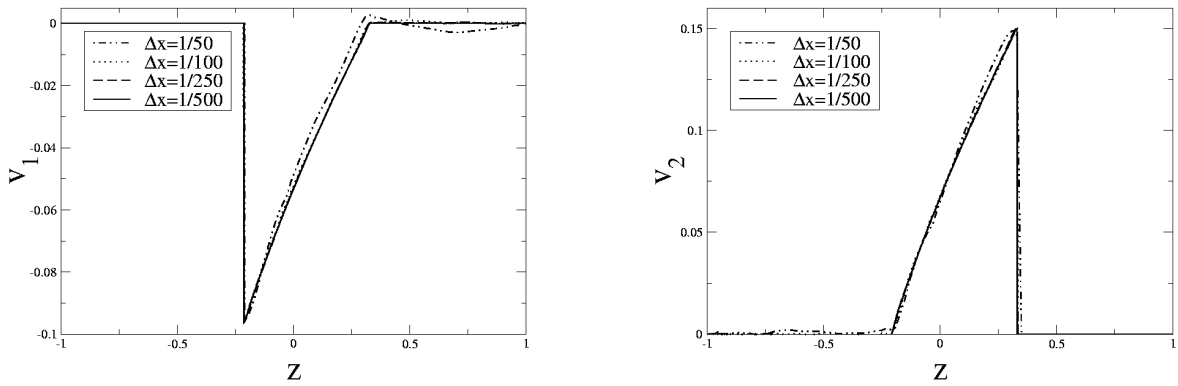

FIG. 10. The convergence of the fluid velocities under mesh refinement. Here $\lambda^{2}=100$ and $t=2.0$. Left frame: light (upper) fluid. Right frame: heavy (lower) fluid.
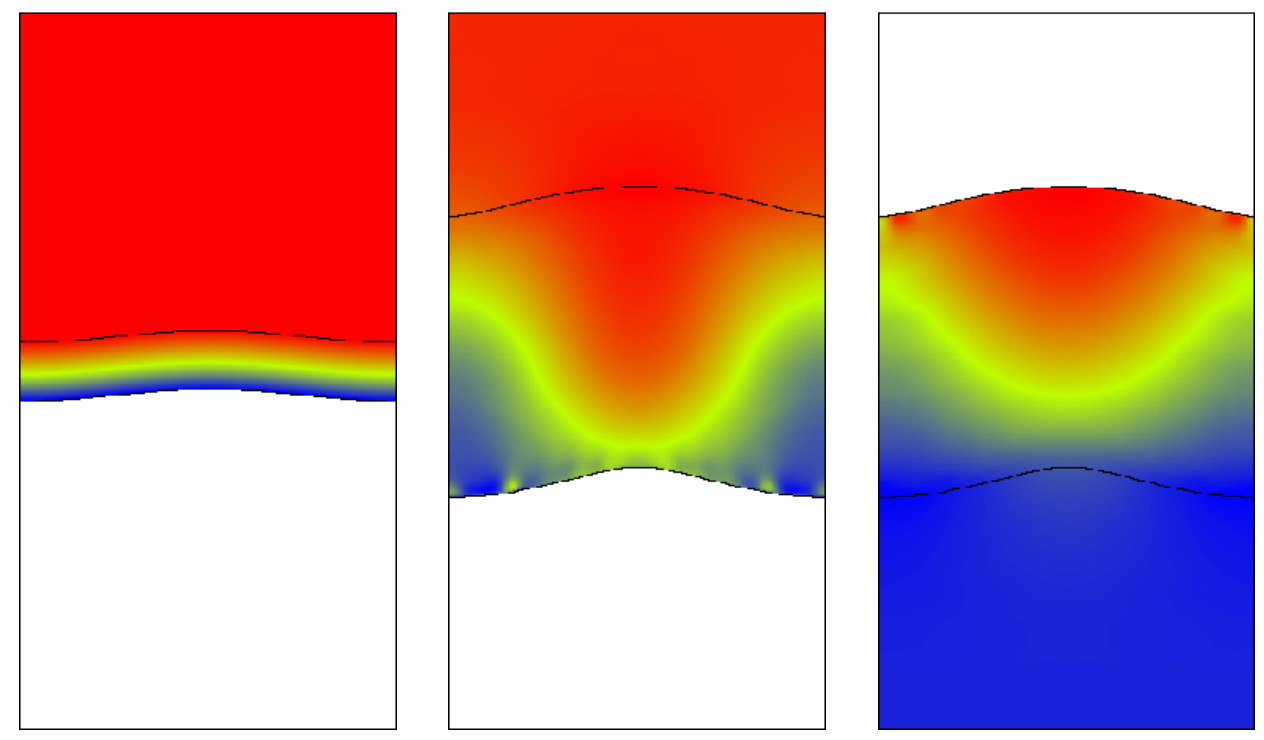

FIG. 11. The fluid velocities of the $2 D$ simulations. Here $\lambda=10$. Left frame: light (upper) fluid velocity $v_{1 y}$ at $t=0$. Center frame: light (upper) fluid velocity $v_{1 y}$ at $t=3.0$. Right frame: heavy (lower) fluid velocity $v_{2 y}$ at $t=3.0$.

mean pressure converge much more rapidly than does the pressure difference.

4.3. 2D simulations. The purpose of multiphase models is to eliminate most of the geometrical complexity through averaging. All fine scale geometrical details associated with complex interface positions are eliminated, and only large scale effects remain. Accordingly, our 2D study is initialized as a single sine wave perturbation to a planar mixing layer. In Figure 11 we present results, plotting $v_{1 y}$ and $v_{2 y}$, in their dependence on the space variables $x, y$. Note that the light fluid is on the top, and so $v_{1 y}$ is undefined in the (lower) region occupied exclusively by the heavy fluid, while 
TABLE 1

Values of $\alpha_{1}$ determined from theory, experiment, and simulation.

\begin{tabular}{|l|l|l|}
\hline \multirow{2}{*}{ Merger models } & Cheng et al. [8, 4] & $\alpha_{1} \approx 0.05-0.06$ \\
\cline { 2 - 3 } & Oron et al. [38] & $\alpha_{1} \approx 0.05$ \\
\hline \multirow{3}{*}{ Experiment } & Read/Youngs [39,43] & $\alpha_{1} \approx 0.063-0.077$ \\
\cline { 2 - 3 } & Dimonte/Schneider [13, 15, 16] & $\alpha_{1}=0.05 \pm 0.01$ \\
\hline \multirow{3}{*}{ Simulation } & Cheng et al./George et al. [5, 19] & $\begin{array}{l}\alpha_{1} \approx 0.07-0.08 \text { (tracked) } \\
\alpha_{1} \approx 0.035 \text { (untracked) }\end{array}$ \\
\cline { 2 - 3 } & & $\alpha_{1} \approx 0.04-0.05$ \\
\cline { 2 - 3 } & Youngs [45] & $\alpha_{1} \approx 0.03$ \\
\cline { 2 - 3 } & Youngs [46] & \multicolumn{2}{|c|}{} \\
\hline
\end{tabular}

$v_{2 y}$ is undefined in the upper region. In the left frame, we display the initial conditions for $v_{1 y}$, defined as a $y$-axis perturbation of a purely planar, 1D initialization, the latter discussed in section 4 . In the center frame, we show $v_{1 y}$ for a later time development of the instability. The influence of the macroscopic perturbation of the interface is evident. All $v_{1 y}$ velocities are negative, the blue being more strongly negative. Thus the light fluid is flowing most strongly into a dip on the lower edge of the mixing zone. The lower edge is the bubble edge. We interpret the macrodisturbance as bubble and spike-type structures on the bubble edge (and also the upper spike edge). In this terminology, the macrolight fluid velocity is flowing into a macrobubble disturbance on the bubble edge of the mixing zone.

In the right frame of Figure 11, we present the later time heavy fluid $v_{2 y}$ velocity. This velocity is everywhere positive and is most strongly positive at the upper edge in the center. Using a similar terminology and analysis to that used for $v_{1 y}$, we can say that the heavy fluid is flowing most strongly into a macrospike-type disturbance of the spike (upper) edge of the mixing zone.

5. Conclusion. We have presented a numerical algorithm for the solution of the chunk mix multiphase flow equations (1) coupled with a phenomenological law (8) for the motion of the edges of the mixing layer. This algorithm extends the front tracking algorithm and deals with issues unique to the front tracking model for this multiphase model. A new analytic solution for the incompressible pressures has been described, thereby completing previous analytic solutions. The numerical algorithm has been validated by comparison to the analytic incompressible limit and by convergence under mesh refinement. The sensitive dependence of the pressure difference on the compressible drag coefficients $C_{k}$ and thus on the edge motions or the mixing coefficients $\alpha_{k}=\alpha_{k}\left(C_{k}\right)$ has been explained in terms of the relation of the exact momentum equation evaluated at the mixing zone edge to the phenomenological buoyancy drag equation. We demonstrate insensitivity of the rest of the solution to these quantities.

Appendix. The bubble growth rate $\alpha_{1}$. Mixing rates for acceleration driven flows have been characterized by experiments, theories, and simulation studies. Read [39] and Youngs [43] have published the result that the bubble acceleration rate $\alpha_{1}$ is almost a constant, with $\alpha_{1} \approx 0.063-0.077$ in three-dimensional (3D) experiments. The experiments have been repeated by various authors and similar values of $\alpha_{1}$ have been obtained; we mention the experiments of Dimonte [13] and Dimonte and Schneider $[15,16]$ giving $\alpha_{1}=0.05 \pm 0.01$. The theoretically determined mixing rates $[8,4,38]$ are obtained from a bubble merger model. They are in agreement with the experiments. 3D simulations [5, 19] of the Rayleigh-Taylor instability, carried to later time, give a growth rate $\alpha_{1} \approx 0.07-0.08$. Only the untracked (TVD) simulations [19] are in significant disagreement; careful analysis points to numerical diffusion at the contact as the cause of this discrepancy [19]. See Table 1. 


\section{REFERENCES}

[1] U. Alon, J. Hecht, D. Mukamel, and D. Shvarts, Scale invariant mixing rates of hydrodynamically unstable interfaces, Phys. Rev. Lett., 72 (1994), pp. 2867-2870.

[2] J. Bell, P. Colella, And J. Trangenstein, Higher order Godunov methods for general systems of hyperbolic conservation laws, J. Comput. Phys., 82 (1989), pp. 362-397.

[3] Y. Chen, J. Glimm, D. Saltz, D. H. Sharp, and Q. Zhang, A two-phase flow formulation for the Rayleigh-Taylor mixing zone and its renormalization group solution, in Proceedings of the Fifth International Workshop on Compressible Turbulent Mixing, R. Young, J. Glimm, and B. Boston, eds., World Scientific, River Edge, NJ, 1996, pp. 23-32.

[4] B. Cheng, J. Glimm, H. Jin, And D. H. Sharp, Theoretical methods for the determination of mixing, Laser and Particle Beams, to appear.

[5] B. Cheng, J. Glimm, X. L. Li, And D. H. Sharp, Subgrid models and DNS studies of fluid mixing, in Proceedings of the 7th International Conference on the Physics of Compressible Turbulent Mixing, St. Petersburg, Russia, 1999, E. Meshkov, Y. Yanilkin, and V. Zhmailo, eds., Sarov, Nizhny Novgorod region, Russia, 2001, pp. 385-390.

[6] B. Cheng, J. Glimm, D. Saltz, and D. H. Sharp, Boundary conditions for a two pressure two phase flow model, Phys. D, 133 (1999), pp. 84-105.

[7] B. Cheng, J. Glimm, And D. H. Sharp, Density dependence of Rayleigh-Taylor and Richtmyer-Meshkov mixing fronts, Phys. Lett. A, 268 (2000), pp. 366-374.

[8] B. Cheng, J. Glimm, And D. H. Sharp, A 3-D RNG bubble merger model for Rayleigh-Taylor mixing, Chaos, 12 (2002), pp. 267-274.

[9] B. Cheng, J. Glimm, and D. H. Sharp, Multi-temperature multiphase flow model, Z. Angew Math. Phys., 53 (2002), pp. 211-238.

[10] I-L. Chern, J. Glimm, O. McBryan, B. Plohr, and S. Yaniv, Front tracking for gas dynamics, J. Comput. Phys., 62 (1986), pp. 83-110.

[11] P. Colella, A direct Eulerian MUSCL scheme for gas dynamics, SIAM J. Sci. Statist. Comput. 6 (1985), pp. 104-117.

[12] M. G. Crandall And A. Majda, The method of fractional steps for conservation laws, Math. Comp., 34 (1980), pp. 285-314.

[13] G. Dimonte, Nonlinear evolution of the Rayleigh-Taylor and Richtmyer-Meshkov instabilities, Phys. Plasmas, 6 (1999), pp. 2009-2015.

[14] G. Dimonte, Spanwise homogeneous buoyancy-drag model for Rayleigh-Taylor mixing and experimental evaluation, Phys. Plasmas, 7 (2000), pp. 2255-2269.

[15] G. Dimonte And M. Schneider, Turbulent Rayleigh-Taylor instability experiments with variable acceleration, Phys. Rev. E, 54 (1996), pp. 3740-3743.

[16] G. Dimonte And M. Schneider, Density ratio dependence of Rayleigh-Taylor mixing for sustained and impulsive acceleration histories, Phys. Fluids, 12 (2000), pp. 304-321.

[17] D. A. Drew, Mathematical modeling of two-phase flow, Ann. Rev. Fluid Mech., 15 (1983), pp. 261-291.

[18] D. EBIN, The motion of slightly compressible fluids viewed as a motion with strong constraining force, Ann. of Math. (2), 105 (1977), pp. 141-200.

[19] E. George, J. Glimm, X. L. Li, A. Marchese, and Z. L. Xu, A comparison of experimental, theoretical, and numerical simulation Rayleigh-Taylor mixing rates, Proc. Natl. Acad. Sci. USA, 99 (2002), pp. 2587-2592.

[20] J. Glimm, J. W. Grove, X. L. Li, W. Oh, And D. H. Sharp, A critical analysis of RayleighTaylor growth rates, J. Comput. Phys., 169 (2001), pp. 652-677.

[21] J. Glimm, J. W. Grove, X. L. Li, K.-M. Shyue, Y. Zeng, and Q. Zhang, Three-dimensional front tracking, SIAM J. Sci. Comput., 19 (1998), pp. 703-727.

[22] J. Glimm, J. W. Grove, X. L. Li, and D. C. Tan, Robust computational algorithms for dynamic interface tracking in three dimensions, SIAM J. Sci. Comput., 21 (2000), pp. $2240-2256$.

[23] J. Glimm, J. W. Grove, X.-L. Li, and N. Zhao, Simple Front Tracking, Contemp. Math. 238, G.-Q. Chen and E. DiBenedetto, eds., AMS, Providence, RI, 1999, pp. 133-149.

[24] J. Glimm And H. Jin, An asymptotic analysis of two-phase fluid mixing, Bol. Soc. Brasil. Mat. (N.S.), 32 (2001), pp. 213-236.

[25] J. Glimm, D. Marchesin, And O. McBryan, Subgrid resolution of fluid discontinuities II, J. Comput. Phys., 37 (1980), pp. 336-354.

[26] J. Glimm, D. Saltz, And D. H. Sharp, A general closure relation for incompressible mixing layers induced by interface instabilities, in Proceedings of the Sixth International Workshop on the Physics of Compressible Turbulent Mixing, Imprimerie Caractère, Marseille, France, 1997, pp. 179-184. 
[27] J. Glimm, D. Saltz, and D. H. Sharp, Statistical evolution of chaotic fluid mixing, Phys. Rev. Lett., 80 (1998), pp. 712-715.

[28] J. Glimm, D. Saltz, and D. H. Sharp, Two-pressure two-phase flow, in Nonlinear Partial Differential Equations, G.-Q. Chen, Y. Li, and X. Zhu, eds., World Scientific, River Edge, NJ, 1998.

[29] J. Glimm, D. SAltz, And D. H. Sharp, Two-phase modeling of a fluid mixing layer, J. Fluid Mech., 378 (1999), pp. 119-143.

[30] J. W. Grove, The interaction of shock waves with fluid interfaces, Adv. Appl. Math., 10 (1989), pp. 201-227.

[31] J. C. V. Hansom, P. A. Rosen, T. J. Goldack, P. Fieldhouse, K. Oades, N. Cowperthwaite, D. L. Youngs, N. Mawhinney, and A. J. Baxter, Radiation driven planar foil instability and mix experiments at the AWE HELEN laser, Laser and Particle Beams, 8 (1990), pp. 51-71.

[32] D. Hoff, The zero-mach limit of compressible flows, Comm. Math. Phys., 192 (1998), pp. $543-554$.

[33] H. Jin, The Incompressible Limit of Compressible Multiphase Flow Equations, Ph.D. thesis, SUNY at Stony Brook, Stony Brook, NY, 2001.

[34] S. KLAinerman AND A. MAJDA, Singular limits of quasilinear hyperbolic systems with large parameters and the incompressible limit of compressible fluids, Comm. Pure Appl. Math., 34 (1981), pp. 481-524.

[35] S. Klainerman and A. Majda, Compressible and incompressible fluids, Comm. Pure Appl. Math., 35 (1982), pp. 629-651.

[36] L. Landau and E. Lifshitz, Fluid Mechanics, Addison-Wesley, Reading, MA, 1959.

[37] P. Lax and B. Wendroff, Systems of conservation laws, Comm. Pure Appl. Math., 13 (1960), pp. 217-237.

[38] D. Oron, L. Arazi, D. Kartoon, A. Rikanati, U. Alon, and D. Shvarts, Dimensionality dependence of the Rayleigh-Taylor and Richtmyer-Meshkov instability late-time scaling laws, Phys. Plasmas, 8 (2001), pp. 2883-2889.

[39] K. I. READ, Experimental investigation of turbulent mixing by Rayleigh-Taylor instability, Phys. D, 12 (1984), pp. 45-58.

[40] R. Richtmyer and K. Morton, Difference Methods for Initial Value Problems, 2nd ed., Interscience, New York, 1967.

[41] S. L. Soo, Particulates and Continuum: Multiphase Fluid Dynamics, Hemisphere, New York, 1989.

[42] G. Strang, Accurate partial difference methods II: Nonlinear problems, Numer. Math., 6 (1964), pp. 37-46.

[43] D. L. Youngs, Numerical simulation of turbulent mixing by Rayleigh-Taylor instability, Phys. D, 12 (1984), pp. 32-44.

[44] D. L. Youngs, Modeling turbulent mixing by Rayleigh-Taylor instability, Phys. D, 37 (1989), pp. 270-287.

[45] D. L. Youngs, Three-dimensional numerical simulation of turbulent mixing by Rayleigh-Taylor instability, Phys. Fluids A, 3 (1991), pp. 1312-1319.

[46] D. L. Youngs, private communication, 1999. 